\title{
Power Series Representations for Complex Bosonic Effective Actions. II. A Small Field Renormalization Group Flow
}

\author{
Tadeusz Balaban \\ Department of Mathematics \\ Rutgers, The State University of New Jersey \\ 110 Frelinghuysen Rd \\ Piscataway, NJ 08854-8019 \\ tbalaban@math.rutgers.edu \\ Joel Feldman \\ Department of Mathematics \\ University of British Columbia \\ Vancouver, B.C. \\ CANADA V6T $1 Z 2$ \\ feldman@math.ubc.ca \\ http://www.math.ubc.ca/ feldman/ \\ Horst Knörrer, Eugene Trubowitz \\ Mathematik \\ ETH-Zentrum \\ CH-8092 Zürich \\ SWITZERLAND \\ knoerrer@math.ethz.ch, trub@math.ethz.ch \\ http://www.math.ethz.ch/ knoerrer/
}

Abstract. In a previous paper, we developed a power series representation and estimates for an effective action of the form

$$
\ln \frac{\int e^{f\left(\alpha_{1}, \cdots, \alpha_{s} ; z^{*}, z\right)} d \mu\left(z^{*}, z\right)}{\int e^{f\left(0, \cdots, 0 ; z^{*}, z\right)} d \mu\left(z^{*}, z\right)}
$$

Here, $f\left(\alpha_{1}, \cdots, \alpha_{s} ; z_{*}, z\right)$ is an analytic function of the complex fields $\alpha_{1}(\mathbf{x}), \cdots, \alpha_{s}(\mathbf{x})$, $z_{*}(\mathbf{x}), z(\mathbf{x})$ indexed by $\mathbf{x}$ in a finite set $X$, and $d \mu\left(z^{*}, z\right)$ is a compactly supported product measure. Such effective actions occur in the small field region for a renormalization group analysis. We illustrate the technique by a model renormalization group flow motivated by the ultraviolet regime in many boson systems. 


\section{Introduction}

Consider the grand canonical partition function, at temperature $T$ and chemical potential $\mu$, for a many boson system moving in a metric space $X$ with a finite number of points and metric $d$. Suppose that the Hamiltonian $H$ is the sum of a single particle operator (for example, the discrete Laplacian) with kernel $\mathrm{h}(\mathbf{x}, \mathbf{y})$ and a two body operator given by a real, symmetric, repulsive pair potential $2 v(\mathbf{x}, \mathbf{y})$.

In $[2$, Theorem 2.2], we proved the functional integral representation

$$
\begin{aligned}
& \operatorname{Tr} e^{-\frac{1}{k T}(H-\mu N)} \\
& =\lim _{\varepsilon \rightarrow 0} \int \prod_{\tau \in \varepsilon \mathbb{Z} \cap\left(0, \frac{1}{k T}\right]}\left[d \tilde{\mu}_{\mathrm{R}_{\varepsilon}}\left(\alpha_{\tau}^{*}, \alpha_{\tau}\right) \zeta_{\varepsilon}\left(\alpha_{\tau-\varepsilon}, \alpha_{\tau}\right) e^{\left\langle\alpha_{\tau-\varepsilon}^{*}, j(\varepsilon) \alpha_{\tau}\right\rangle-\varepsilon\left\langle\alpha_{\tau-\varepsilon}^{*} \alpha_{\tau} v \alpha_{\tau-\varepsilon}^{*} \alpha_{\tau}\right\rangle}\right]
\end{aligned}
$$

for the partition function, under the convention that $\alpha_{0}=\alpha_{\frac{1}{k T}}$ and the limit $\varepsilon \rightarrow 0$ is restricted to $\varepsilon$ 's dividing $\frac{1}{k T}$ (that is, $\frac{1}{\varepsilon} \in(k T) \mathbb{N}$ ). Here, $N$ is the number operator and, for any $r>0$,

$$
d \tilde{\mu}_{r}\left(\alpha^{*}, \alpha\right)=\prod_{\mathbf{x} \in X} \frac{d \alpha^{*}(\mathbf{x}) \wedge d \alpha(\mathbf{x})}{2 \pi \imath} e^{-\alpha^{*}(\mathbf{x}) \alpha(\mathbf{x})} \chi(|\alpha(\mathbf{x})|<r)
$$

denotes the unnormalised Gaussian measure, cut off at radius $r$, and $\zeta_{\varepsilon}(\alpha, \beta)$ is the characteristic function of

$$
\left\{\alpha, \beta: X \rightarrow \mathbb{C} \mid\|\alpha-\beta\|_{\infty}<p_{0}(\varepsilon)\right\}
$$

The cutoffs $\mathrm{R}_{\varepsilon}$ and $p_{0}(\varepsilon)$ grow at an appropriate rate as $\varepsilon \rightarrow 0 .{ }^{(1)}$ Furthermore, for any $\varepsilon>0$, the operator $j(\varepsilon)=e^{-\varepsilon(\mathrm{h}-\mu)}$. We write the $(\mathbb{R}$-style) scalar product, $\langle f, g\rangle=$ $\sum_{\mathbf{x} \in X} f(\mathbf{x}) g(\mathbf{x})$ for any two fields $f, g: X \rightarrow \mathbb{C}$. $^{(2)}$

The representation (I.1) is the first part of a program to resolve the mathematical difficulties inherent in the time-ultraviolet limit of the formal, coherent state functional integral representation for the partition function and correlation functions of many boson systems. See [5, 2.66] and the discussion in the introduction to [1]. In [4] this program is completed, using techniques of renormalization group analysis. This paper is a description of the "small field part" of that construction.

In [4] we obtain a representation of the functional integral of (I.1) which can be used to analyze infrared problems. We do so by applying a simple version of a renormalization

(1) One can think of $\mathrm{R}_{\varepsilon}$ as growing a bit faster than $\frac{1}{\sqrt[4]{\varepsilon}}$ and of $p_{0}(\varepsilon)$ as a power of $\ln \frac{1}{\varepsilon}$ or a very small power of $\frac{1}{\varepsilon}$.

(2) Thus the usual scalar product over $\mathbb{C}^{|X|}$ is $\left\langle f^{*}, g\right\rangle$. 
group procedure, namely "decimation". In each decimation step we integrate out every "second" variable. In the first step, we integrate out $\alpha_{\tau^{\prime}}$ with $\tau^{\prime}=\varepsilon, 3 \varepsilon, 5 \varepsilon, \cdots$. The integral with respect to these variables factorizes into the product, over $\tau=2 \varepsilon, 4 \varepsilon, 6 \varepsilon, \cdots$, of the independent integrals

$$
\begin{array}{r}
\int d \tilde{\mu}_{\mathrm{R}_{\varepsilon}}\left(\alpha_{\tau-\varepsilon}^{*}, \alpha_{\tau-\varepsilon}\right) \zeta_{\varepsilon}\left(\alpha_{\tau-2 \varepsilon}, \alpha_{\tau-\varepsilon}\right) \\
e^{\left\langle\alpha_{\tau-2 \varepsilon}^{*}, j(\varepsilon) \alpha_{\tau-\varepsilon}\right\rangle-\varepsilon\left\langle\alpha_{\tau-2 \varepsilon}^{*} \alpha_{\tau-\varepsilon} v \alpha_{\tau-2 \varepsilon}^{*} \alpha_{\tau-\varepsilon}\right\rangle} \\
e^{\left\langle\alpha_{\tau-\varepsilon}^{*}, j(\varepsilon) \alpha_{\tau}\right\rangle-\varepsilon\left\langle\alpha_{\tau-\varepsilon}^{*} \alpha_{\tau}, v \alpha_{\tau-\varepsilon}^{*} \alpha_{\tau}\right\rangle} \zeta_{\varepsilon}\left(\alpha_{\tau-\varepsilon}, \alpha_{\tau}\right)
\end{array}
$$

That is, assuming that $\frac{1}{k T} \in 2 \varepsilon \mathbb{N}$,

$$
\begin{array}{r}
\int \prod_{\tau \in \varepsilon \mathbb{Z} \cap\left(0, \frac{1}{k T}\right]}\left[d \tilde{\mu}_{\mathrm{R}_{\varepsilon}}\left(\alpha_{\tau}^{*}, \alpha_{\tau}\right) \zeta_{\varepsilon}\left(\alpha_{\tau-\varepsilon}, \alpha_{\tau}\right) e^{\left\langle\alpha_{\tau-\varepsilon}^{*}, j(\varepsilon) \alpha_{\tau}\right\rangle-\varepsilon\left\langle\alpha_{\tau-\varepsilon}^{*} \alpha_{\tau} v \alpha_{\tau-\varepsilon}^{*} \alpha_{\tau}\right\rangle}\right] \\
=\int_{\tau \in 2 \varepsilon \mathbb{Z} \cap\left(0, \frac{1}{k T}\right]} \prod_{\mathrm{R}_{\varepsilon}}\left(\alpha_{\tau}^{*}, \alpha_{\tau}\right) I_{1}\left(\varepsilon ; \alpha_{\tau-2 \varepsilon}^{*}, \alpha_{\tau}\right)
\end{array}
$$

where

$$
\begin{aligned}
& I_{1}\left(\varepsilon ; \alpha^{*}, \beta\right)=\int d \tilde{\mu}_{\mathrm{R}_{\varepsilon}}\left(\phi^{*}, \phi\right) \zeta_{\varepsilon}(\alpha, \phi) e^{\left\langle\alpha^{*}, j(\varepsilon) \phi\right\rangle+\left\langle\phi^{*}, j(\varepsilon) \beta\right\rangle} \\
& \quad e^{-\varepsilon\left(\left\langle\alpha^{*} \phi, v \alpha^{*} \phi\right\rangle+\left\langle\phi^{*} \beta, v \phi^{*} \beta\right\rangle\right)} \zeta_{\varepsilon}(\phi, \beta)
\end{aligned}
$$

After $n-1$ additional decimation steps we will have integrated out those $\alpha_{\tau}$ 's with $\tau \in\left(\varepsilon \mathbb{Z} \backslash\left(2^{n} \varepsilon\right) \mathbb{Z}\right) \cap\left(0, \frac{1}{k T}\right]$ leaving an integrand which is a function of the $\alpha_{\tau}$ 's with $\tau \in\left(2^{n} \varepsilon\right) \mathbb{Z} \cap\left[0, \frac{1}{k T}\right]$. Thus, for $\frac{1}{k T} \in 2^{n} \varepsilon \mathbb{N}$, we write

$$
\begin{array}{r}
\int \prod_{\tau \in \varepsilon \mathbb{Z} \cap\left(0, \frac{1}{k T}\right]}\left[d \tilde{\mu}_{\mathrm{R}_{\varepsilon}}\left(\alpha_{\tau}^{*}, \alpha_{\tau}\right) \zeta_{\varepsilon}\left(\alpha_{\tau-\varepsilon}, \alpha_{\tau}\right) e^{\left.\left\langle\alpha_{\tau-\varepsilon}^{*}, j(\varepsilon) \alpha_{\tau}\right\rangle-\varepsilon\left\langle\alpha_{\tau-\varepsilon}^{*} \alpha_{\tau} v \alpha_{\tau-\varepsilon}^{*} \alpha_{\tau}\right\rangle\right]}\right. \\
=\int \prod_{\tau \in\left(2^{n} \varepsilon\right) \mathbb{Z} \cap\left(0, \frac{1}{k T}\right]} d \tilde{\mu}_{\mathrm{R}_{\varepsilon}}\left(\alpha_{\tau}^{*}, \alpha_{\tau}\right) I_{n}\left(\varepsilon, \alpha_{\tau-2^{n} \varepsilon}^{*}, \alpha_{\tau}\right)
\end{array}
$$

where the functions $I_{n}\left(\varepsilon ; \alpha^{*}, \beta\right)$ are recursively defined by (I.2) and

$$
I_{n+1}\left(\varepsilon ; \alpha^{*}, \beta\right)=\int d \tilde{\mu}_{\mathrm{R}_{\varepsilon}}\left(\phi^{*}, \phi\right) I_{n}\left(\varepsilon ; \alpha^{*}, \phi\right) I_{n}\left(\varepsilon ; \phi^{*}, \beta\right)
$$

The main result of [4] is the construction and description of a functional $I_{\theta}\left(\alpha^{*}, \beta\right)$, defined for $\theta \in(0, \Theta]$ (where $\Theta=O(1)$, independent of $v$ ), such that

$$
I_{\theta}\left(\alpha^{*}, \beta\right)=\lim _{m \rightarrow \infty} I_{m}\left(2^{-m} \theta ; \alpha^{*}, \beta\right)
$$


Then, for any $p$ such that $\frac{1}{p k T} \in(0, \Theta]$

$$
\operatorname{Tr} e^{-\frac{1}{k T}(H-\mu N)}=\int \prod_{n=1}^{p}\left[\prod_{\mathbf{x} \in X} \frac{d \phi_{n}(\mathbf{x})^{*} \phi_{n}(\mathbf{x})}{2 \pi \imath} e^{-\phi_{n}(\mathbf{x})^{*} \phi_{n}(\mathbf{x})}\right] I_{\frac{1}{p k T}}\left(\phi_{n-1}^{*}, \phi_{n}\right)
$$

with the convention $\phi_{0}=\phi_{p}$. (I.5) can be the starting point for an infrared analysis.

In [4] we describe the functions $I_{n}\left(\varepsilon ; \alpha^{*}, \beta\right)$ and $I_{\theta}\left(\alpha^{*}, \beta\right)$ as sums over "large/small field decompositions" of $X$. The dominant part in the large/small field decomposition is called the "pure small field part" and is obtained by replacing the full integrals (I.4) with integrals over appropriate neighbourhoods of stationary points. See $\S I I$.

In this note, we discuss a toy model, which we call the "stationary phase approximation" (SP), in which all domains of integration are restricted, simply by fiat, to neighbourhoods of stationary points. These neighbourhoods will be measured by radii $r(\delta)$ where $\delta \mapsto r(\delta)$ is a positive and monotonically decreasing function. We now give a description of the stationary phase approximation and derive estimates for it. To do this, introduce the notation $\varepsilon_{n}=2^{n} \varepsilon$ and

$$
\mathcal{V}_{\delta}\left(\varepsilon ; \alpha^{*}, \beta\right)=-\varepsilon \sum_{\tau \in \varepsilon \mathbb{Z} \cap[0, \delta)}\left\langle\left[j(\tau) \alpha^{*}\right][j(\delta-\tau-\varepsilon) \beta], v\left[j(\tau) \alpha^{*}\right][j(\delta-\tau-\varepsilon) \beta]\right\rangle
$$

It will turn out that there is a function $\mathcal{E}_{\delta}\left(\varepsilon ; \alpha^{*}, \beta\right)$ of the fields $\alpha^{*}, \beta$ with the property

$$
I_{n}^{(\mathrm{SP})}\left(\varepsilon ; \alpha^{*}, \beta\right)=\mathcal{Z}_{\varepsilon_{n}}(\varepsilon)^{|X|} e^{\left\langle\alpha^{*}, j\left(\varepsilon_{n}\right) \beta\right\rangle+\mathcal{V}_{\varepsilon_{n}}\left(\varepsilon ; \alpha^{*}, \beta\right)+\mathcal{E}_{\varepsilon_{n}}\left(\varepsilon ; \alpha^{*}, \beta\right)}
$$

The function $\mathcal{E}_{\delta}\left(\varepsilon ; \alpha^{*}, \beta\right)$ is defined for real numbers $0<\varepsilon \leq \delta \leq \Theta$ such that $\delta=2^{n} \varepsilon$ for some integer $n \geq 0$. The normalization constant $\mathcal{Z}_{\delta}(\varepsilon)$ is defined in Appendix $\mathrm{C}$. It is chosen so that $\mathcal{E}_{\delta}(\varepsilon ; 0,0)=0$. It is extremely close to 1 . The "irrelevant" contributions $\mathcal{E}_{\delta}\left(\varepsilon ; \alpha^{*}, \beta\right)$ to the effective action are characterized by the recursion relation

$$
\begin{aligned}
\mathcal{E}_{\varepsilon}\left(\varepsilon ; \alpha^{*}, \beta\right) & =0 \\
\mathcal{E}_{2 \delta}\left(\varepsilon ; \alpha^{*}, \beta\right) & =\mathcal{E}_{\delta}\left(\varepsilon ; \alpha^{*}, j(\delta) \beta\right)+\mathcal{E}_{\delta}\left(\varepsilon ; j(\delta) \alpha^{*}, \beta\right)+\log \frac{\int d \tilde{\mu}_{r(\delta)}\left(z^{*}, z\right) e^{\partial \mathcal{A}_{\delta}\left(\varepsilon ; \alpha^{*}, \beta ; z^{*}, z\right)}}{\int d \tilde{\mu}_{r(\delta)}\left(z^{*}, z\right)}
\end{aligned}
$$

where

$$
\begin{aligned}
\partial \mathcal{A}_{\delta}\left(\varepsilon ; \alpha^{*}, \beta ; z_{*}, z\right)= & {\left[\mathcal{V}_{\delta}\left(\varepsilon ; \alpha^{*}, j(\delta) \beta+z\right)-\mathcal{V}_{\delta}\left(\varepsilon ; \alpha^{*}, j(\delta) \beta\right)\right] } \\
& +\left[\mathcal{V}_{\delta}\left(\varepsilon ; j(\delta) \alpha^{*}+z_{*}, \beta\right)-\mathcal{V}_{\delta}\left(\varepsilon ; j(\delta) \alpha^{*}, \beta\right)\right] \\
& +\left[\mathcal{E}_{\delta}\left(\varepsilon ; \alpha^{*}, j(\delta) \beta+z\right)-\mathcal{E}_{\delta}\left(\varepsilon ; \alpha^{*}, j(\delta) \beta\right)\right] \\
& +\left[\mathcal{E}_{\delta}\left(\varepsilon ; j(\delta) \alpha^{*}+z_{*}, \beta\right)-\mathcal{E}_{\delta}\left(\varepsilon ; j(\delta) \alpha^{*}, \beta\right)\right]
\end{aligned}
$$

The motivation for this recursion relation comes from a stationary phase construction and is given in the next section. 
We estimate $\mathcal{E}_{\delta}\left(\varepsilon ; \alpha^{*}, \beta\right)$ in terms of norms as in [3, Definition 2.6 and, more specifically, (A.1)]. Assume that $X$ is a metric space. Choose a constant $\mathrm{m} \geq 0$ as a spatial exponential decay rate, and a positive monotonically decreasing function $\delta \mapsto \kappa(\delta)$ to measure the radius of convergence of the expansion of $\mathcal{E}_{\delta}\left(\varepsilon ; \alpha^{*}, \beta\right)$ in powers of the fields $\alpha^{*}$ and $\beta$.

We define the norm of the power series

$$
f\left(\alpha^{*}, \beta\right)=\sum_{k, \ell \geq 0} \sum_{\substack{\mathbf{x}_{1}, \cdots, \mathbf{x}_{k} \in X \\ \mathbf{y}_{1}, \cdots, \mathbf{y}_{\ell} \in X}} a\left(\mathbf{x}_{1}, \cdots, \mathbf{x}_{k} ; \mathbf{y}_{1}, \cdots, \mathbf{y}_{\ell}\right) \alpha\left(\mathbf{x}_{1}\right)^{*} \cdots \alpha\left(\mathbf{x}_{k}\right)^{*} \beta\left(\mathbf{y}_{1}\right) \cdots \beta\left(\mathbf{y}_{\ell}\right)
$$

(with the coefficients $a\left(\mathbf{x}_{1}, \cdots, \mathbf{x}_{k} ; \mathbf{y}_{1}, \cdots, \mathbf{y}_{\ell}\right)$ invariant under permutations of $\mathbf{x}_{1}, \cdots, \mathbf{x}_{k}$ and of $\left.\mathbf{y}_{1}, \cdots, \mathbf{y}_{\ell}\right)$ to be

$$
\left\|f\left(\alpha^{*}, \beta\right)\right\|_{\delta}=\sum_{k, \ell \geq 0} \max _{\mathbf{x} \in X} \max _{1 \leq i \leq k+\ell} \sum_{\substack{(\overrightarrow{\mathbf{x}}, \overrightarrow{\mathbf{y}}) \in X^{k} \times X^{\ell} \\(\overrightarrow{\mathbf{x}}, \overrightarrow{\mathbf{y}})_{i}=\mathbf{x}}} w_{\delta}(\overrightarrow{\mathbf{x}} ; \overrightarrow{\mathbf{y}})|a(\overrightarrow{\mathbf{x}} ; \overrightarrow{\mathbf{y}})|
$$

with the weight system

$$
w_{\delta}(\overrightarrow{\mathbf{x}} ; \overrightarrow{\mathbf{y}})=\kappa(\delta)^{k+\ell} e^{\mathrm{m} \tau(\overrightarrow{\mathbf{x}}, \overrightarrow{\mathbf{y}})} \quad \text { for }(\overrightarrow{\mathbf{x}}, \overrightarrow{\mathbf{y}}) \in X^{k} \times X^{\ell}
$$

where $\tau(\overrightarrow{\mathbf{x}}, \overrightarrow{\mathbf{y}})$ is the minimal length of a tree which contains vertices at the points of the set $\left\{\mathbf{x}_{1}, \cdots, \mathbf{x}_{k}, \mathbf{y}_{1}, \cdots, \mathbf{y}_{\ell}\right\}$. In the language of [3, Definitions 2.5 and 2.6], $w_{\delta}$ is the weight system with metric $\mathrm{m} d$ that associates the constant weight factor $\kappa(\delta)$ to the fields $\alpha^{*}$ and $\beta$ and the norm $\left\|f\left(\alpha^{*}, \beta\right)\right\|_{\delta}$ is denoted $\left\|f\left(\alpha^{*}, \beta\right)\right\|_{w_{\delta}}$. For any operator $\mathcal{A}$ on $\mathbb{C}^{X}$, with kernel $\mathcal{A}(\mathbf{x}, \mathbf{y})$, we define the weighted $L^{1}-L^{\infty}$ operator norm

$$
\|\mathcal{A} \mid\|=\max \left\{\sup _{\mathbf{x} \in X} \sum_{\mathbf{y} \in X} e^{\operatorname{md} d(\mathbf{x}, \mathbf{y})}|\mathcal{A}(\mathbf{x}, \mathbf{y})|, \sup _{\mathbf{y} \in X} \sum_{\mathbf{x} \in X} e^{\operatorname{md}(\mathbf{x}, \mathbf{y})}|\mathcal{A}(\mathbf{x}, \mathbf{y})|\right\}
$$

as in [3, Definition A.1].

The quantities relevant for the estimates of $\mathcal{E}_{\delta}\left(\varepsilon ; \alpha^{*}, \beta\right)$, in addition to the radii $r(\delta)$ and $\kappa(\delta)$, are the norm $\|v\| \|$ of the interaction, a constant $K_{j}$ such

$$
\|j(\tau)\| \leq e^{K_{j} \tau} \quad \text { and } \quad\|j(\tau)-\mathbb{1}\| \| \leq K_{j} \tau e^{K_{j} \tau} \quad \text { for } \tau \geq 0
$$

(see Corollary B.2) and a constant $0<\Theta \leq 1$ that bounds the range for which the constructions work. On these quantities we make the

Hypothesis I.1 We assume that the monotonically decreasing functions $r(t)$ and $\kappa(t)$ do not decrease too quickly. Precisely, 
(i) $1 \leq r(t) \leq 2 r(2 t)$ and $1 \leq \kappa(t) \leq 2 \kappa(2 t)$, for all $0 \leq t \leq \Theta / 2$,

On the other hand, $r(t)$ and $\kappa(t)$ must decrease quickly enough and $r(t)$ must be sufficiently small compared to $\kappa(t)$ that

(ii) $e^{t K_{j}} \frac{\kappa(2 t)}{\kappa(t)}+4 \frac{r(t)}{\kappa(t)} \leq 1$, for all $0 \leq t \leq \Theta / 2$,

(iii) $r(t)[r(t)-r(2 t)] \geq 2$, for all $0 \leq t \leq \Theta / 2$

We also assume that there are constants $K_{E}$ and $q \geq 1$ such that

(iv) $t\|v\| \| r(t) \kappa(t)^{3} \leq \frac{1}{K_{E}}$, for all $0 \leq t \leq \Theta$,

(v) $\frac{1}{C\left(\Theta, K_{E}\right)} \frac{2}{q} \leq\left(\frac{\kappa(t)}{\kappa(2 t)}\right)^{4} \leq C\left(\Theta, K_{E}\right) 4\left(\frac{r(2 t)}{r(t)}\right)^{4}$, for all $0 \leq t \leq \Theta / 2$, where $C\left(\Theta, K_{E}\right)=e^{-4 \Theta K_{j}}\left[1-\frac{2^{33} e^{14 K_{j}}}{K_{E}}\right]$

and

(vi) $t^{2} \sum_{k=0}^{\infty}\left(\frac{q}{4}\right)^{k} r\left(\frac{t}{2^{k}}\right)^{2} \kappa\left(\frac{t}{2^{k}}\right)^{6}$ converges uniformly in $0 \leq t \leq \Theta$

Example I.2 Let $\mathfrak{v}>0$.

(i) Suppose that

$$
\kappa(t)=\frac{1}{\sqrt[4]{\|v\| \|}}\left(\frac{1}{t}\right)^{a_{\kappa}} \quad \text { and } \quad r(t)=\frac{1}{\sqrt[4]{\|v\| \|}}\left(\frac{1}{t}\right)^{a_{r}}
$$

for some constants $0<a_{r}<a_{\kappa}$ obeying $3 a_{\kappa}+a_{r}<1$. We prove in Appendix D that there are constants $K_{E}, \Theta$ and $q$ such that Hypothesis I.1 is fulfilled for all nonzero $v$ with $\|v\| \mid \leq \mathfrak{v}$.

(ii) Suppose that

$$
\kappa(t)=\frac{1}{\sqrt[4]{t\|v v\|}}\left(\ln \frac{1}{t\|v\| \|}\right)^{b} \quad \text { and } \quad r(t)=\left(\ln \frac{1}{t\|v\| \|}\right)^{b}
$$

for some $b \geq 1$. Again, we prove in Appendix D that there are constants $K_{E}, \Theta$ and $q$ such that Hypothesis I.1 is fulfilled for all nonzero $v$ with $\|v\| \| \mathfrak{v}$.

We choose the $p_{0}(\varepsilon)$ of the functional integral representation (I.1) to be $r(\varepsilon)$.

Theorem I.3 Under Hypothesis I.1

$$
\left\|\mathcal{E}_{\delta}\left(\varepsilon ; \alpha^{*}, \beta\right)\right\|_{\delta} \leq K_{E} \delta^{2}\|v\|^{2} r(\delta)^{2} \kappa(\delta)^{6}
$$

for all $0 \leq \varepsilon \leq \delta \leq \Theta$ for which $\frac{\delta}{\varepsilon}$ is a power of 2 . The function $\mathcal{E}_{\delta}\left(\varepsilon ; \alpha^{*}, \beta\right)$ has degree at least two both ${ }^{(3)}$ in $\alpha^{*}$ and $\beta$.

(3) By this we mean that every monomial appearing in its power series expansion contains a factor of the form $\alpha^{*}\left(\mathbf{x}_{1}\right) \alpha^{*}\left(\mathbf{x}_{2}\right) \beta\left(\mathbf{x}_{3}\right) \beta\left(\mathbf{x}_{4}\right)$. 
Theorem I.3 is proven after Proposition III.3. The Theorem is proven by induction on $n$, where $\delta=2^{n} \varepsilon$. The induction step is prepared by Proposition III.3, which is proven using the results of [3]. After the proof of Theorem I.3, we give the proof of

Theorem I.4 The limit

$$
\mathcal{E}_{\theta}\left(\alpha^{*}, \beta\right)=\lim _{m \rightarrow \infty} \mathcal{E}_{\theta}\left(2^{-m} \theta ; \alpha^{*}, \beta\right)
$$

exists uniformly in $0 \leq \theta \leq \Theta$. It fulfills the estimate

$$
\left\|\mathcal{E}_{\theta}\left(\alpha^{*}, \beta\right)\right\|_{\theta} \leq K_{E} \theta^{2}\|v\|^{2} r(\theta)^{2} \kappa(\theta)^{6}
$$

and has degree at least two in both $\alpha^{*}$ and $\beta$.

To take the limit $\varepsilon \rightarrow 0$ in (I.7) observe that, for any fixed $\delta$

$$
\lim _{n \rightarrow \infty} \mathcal{V}_{\delta}\left(2^{-n} \delta ; \alpha^{*}, \beta\right)=\mathcal{V}_{\delta}\left(\alpha^{*}, \beta\right)
$$

where

$$
\mathcal{V}_{\delta}\left(\alpha^{*}, \beta\right)=-\int_{0}^{\delta}\left\langle\left[j(t) \alpha^{*}\right][j(\delta-t) \beta], v\left[j(t) \alpha^{*}\right][j(\delta-t) \beta]\right\rangle d t
$$

The stationary phase approximation to the $I_{\theta}\left(\alpha^{*}, \beta\right)$ constructed in [4] then is

$$
I_{\theta}^{(\mathrm{SP})}\left(\alpha^{*}, \beta\right)=\lim _{m \rightarrow \infty} I_{m}^{(\mathrm{SP})}\left(2^{-m} \theta ; \alpha^{*}, \beta\right)=\mathcal{Z}_{\theta}^{|X|} e^{\left\langle\alpha^{*}, j(\theta) \beta\right\rangle+\mathcal{V}_{\theta}\left(\alpha^{*}, \beta\right)+\mathcal{E}_{\theta}\left(\alpha^{*}, \beta\right)}
$$

The existence of $\mathcal{Z}_{\theta}=\lim _{m \rightarrow \infty} \mathcal{Z}_{\theta}\left(\frac{\theta}{2^{m}}\right)$ is proven in Lemma C.1. 


\section{Stationary Phase and Stokes' Theorem}

To motivate the recursive definition (I.8) of $\mathcal{E}_{\delta}\left(\varepsilon ; \alpha^{*}, \beta\right)$ we replace $I_{n}$ by $I_{n}^{(\mathrm{SP})}$ in the recursion relation (I.4). Inserting (I.7), the resulting integral

$$
\begin{aligned}
& \int d \tilde{\mu}_{\mathrm{R}_{\varepsilon}}\left(\phi^{*}, \phi\right) I_{n}^{(\mathrm{SP})}\left(\varepsilon ; \alpha^{*}, \phi\right) I_{n}^{(\mathrm{SP})}\left(\varepsilon ; \phi^{*}, \beta\right) \\
& =\mathcal{Z}_{n}^{|2 X|} \int d \tilde{\mu}_{\mathrm{R}_{\varepsilon}}\left(\phi^{*}, \phi\right) e^{\left\langle\alpha^{*}, j\left(\varepsilon_{n}\right) \phi\right\rangle+\left\langle\phi^{*}, j\left(\varepsilon_{n}\right) \beta\right\rangle} e^{\mathcal{V}_{\varepsilon_{n}}\left(\varepsilon ; \alpha^{*}, \phi\right)+\mathcal{V}_{\varepsilon_{n}}\left(\varepsilon ; \phi^{*}, \beta\right)} e^{\mathcal{E}_{\varepsilon_{n}}\left(\varepsilon ; \alpha^{*}, \phi\right)+\mathcal{E}_{\varepsilon_{n}}\left(\varepsilon ; \phi^{*}, \beta\right)} \\
& =\mathcal{Z}_{n}^{2|X|} \int\left[\prod_{\mathbf{x} \in X} \frac{d \phi^{*}(\mathbf{x}) \wedge d \phi(\mathbf{x})}{2 \pi \imath} \chi\left(|\phi(\mathbf{x})|<R_{\varepsilon}\right)\right] e^{\mathcal{A}\left(\alpha^{*}, \beta ; \phi^{*}, \phi\right)}
\end{aligned}
$$

with $\mathcal{Z}_{n}=\mathcal{Z}_{\varepsilon_{n}}(\varepsilon)$ and

$$
\begin{aligned}
\mathcal{A}\left(\alpha^{*}, \beta ; \phi_{*}, \phi\right)= & -\left\langle\phi_{*}, \phi\right\rangle+\left\langle\alpha^{*}, j\left(\varepsilon_{n}\right) \phi\right\rangle+\left\langle\phi_{*}, j\left(\varepsilon_{n}\right) \beta\right\rangle \\
& +\mathcal{V}_{\varepsilon_{n}}\left(\varepsilon ; \alpha^{*}, \phi\right)+\mathcal{V}_{\varepsilon_{n}}\left(\varepsilon ; \phi_{*}, \beta\right)+\mathcal{E}_{\varepsilon_{n}}\left(\varepsilon ; \alpha^{*}, \phi\right)+\mathcal{E}_{\varepsilon_{n}}\left(\varepsilon ; \phi_{*}, \beta\right)
\end{aligned}
$$

Here we have written $\mathcal{A}$ as a function of four independent complex fields $\alpha^{*}, \beta, \phi_{*}$ and $\phi$. The activity in (II.1) is obtained by evaluating $\mathcal{A}\left(\alpha^{*}, \beta ; \phi_{*}, \phi\right)$ with $\phi_{*}=\phi^{*}$, the complex conjugate of $\phi$. The reason for introducing independent complex fields $\phi_{*}$ and $\phi$ lies in the fact that the critical point (with respect to the variables $\phi_{*}, \phi$ ) of the quadratic part

$$
-\left\langle\phi_{*}, \phi\right\rangle+\left\langle j\left(\varepsilon_{n}\right) \alpha^{*}, \phi\right\rangle+\left\langle\phi_{*}, j\left(\varepsilon_{n}\right) \beta\right\rangle=-\left\langle\phi_{*}-j\left(\varepsilon_{n}\right) \alpha^{*}, \phi-j\left(\varepsilon_{n}\right) \beta\right\rangle+\left\langle\alpha^{*}, j\left(\varepsilon_{n+1}\right) \beta\right\rangle
$$

of $\mathcal{A}$ is "not real". Precisely, the critical point is

$$
\phi_{*}^{\text {crit }}=j\left(\varepsilon_{n}\right) \alpha^{*}, \quad \phi^{\text {crit }}=j\left(\varepsilon_{n}\right) \beta
$$

and in general $\left(\phi_{*}^{\text {crit }}\right)^{*} \neq \phi^{\text {crit }}$. To do stationary phase, we make the substitution

$$
\phi_{*}=\phi_{*}^{\text {crit }}+z_{*}, \quad \phi=\phi^{\text {crit }}+z
$$

with "fluctuation fields" $z_{*}, z$. With this substitution, the quadratic part of $\mathcal{A}$ is equal to $-\left\langle z_{*}, z\right\rangle+\left\langle\alpha^{*}, j\left(\varepsilon_{n+1}\right) \beta\right\rangle$. So (II.1) becomes

$$
\mathcal{Z}_{n}^{2|X|} e^{\left\langle\alpha^{*}, j\left(\varepsilon_{n+1}\right) \beta\right\rangle}\left[\prod_{\mathbf{x} \in X} \int_{M(\mathbf{x})} \frac{d z_{*}(\mathbf{x}) \wedge d z(\mathbf{x})}{2 \pi \imath} e^{-z_{*}(\mathbf{x}) z(\mathbf{x})}\right] e^{\tilde{\mathcal{A}}\left(\alpha^{*}, \beta ; z_{*}, z\right)}
$$

where

$$
\begin{aligned}
\tilde{\mathcal{A}}\left(\alpha^{*}, \beta ; z_{*}, z\right)= & \mathcal{V}_{\varepsilon_{n}}\left(\varepsilon ; \alpha^{*}, \phi^{\mathrm{crit}}+z\right)+\mathcal{V}_{\varepsilon_{n}}\left(\varepsilon ; \phi_{*}^{\mathrm{crit}}+z_{*}, \beta\right) \\
& +\mathcal{E}_{\varepsilon_{n}}\left(\varepsilon ; \alpha^{*}, \phi^{\mathrm{crit}}+z\right)+\mathcal{E}_{\varepsilon_{n}}\left(\varepsilon ; \phi_{*}^{\text {crit }}+z_{*}, \beta\right) \\
M(\mathbf{x})=\left\{\left(z_{*}(\mathbf{x}), z(\mathbf{x})\right) \mid\left(\phi_{*}^{\mathrm{crit}}(\mathbf{x})+z_{*}(\mathbf{x})\right)^{*}=\phi^{\mathrm{crit}}(\mathbf{x})+z(\mathbf{x})\right. & \text { and } \left.\left|\phi^{\mathrm{crit}}(\mathbf{x})+z(\mathbf{x})\right|<R_{\varepsilon}\right\}
\end{aligned}
$$


(II.3) is the integral over a real $2|X|$ dimensional subset in the complex $2|X|$ dimensional space of fields $z_{*}, z$.

The first step in the stationary phase approximation is to replace, for each $\mathbf{x} \in X$, the set $M(\mathbf{x})$ in (II.3) by the neighbourhood

$$
\begin{aligned}
D(\mathbf{x})=\left\{\left(z_{*}(\mathbf{x}), z(\mathbf{x})\right) \in \mathbb{C}^{2} \mid\right. & \left|z_{*}(\mathbf{x})\right| \leq r\left(\varepsilon_{n}\right),|z(\mathbf{x})| \leq r\left(\varepsilon_{n}\right) \\
& \left.\left(z_{*}(\mathbf{x})+\phi_{*}^{\text {crit }}(\mathbf{x})\right)^{*}=z(\mathbf{x})+\phi^{\text {crit }}(\mathbf{x})\right\}
\end{aligned}
$$

of the critical point. In $[4, \S \mathrm{VI}]$ we show that the error introduced by this approximation is extremely small (even when $D(\mathbf{x})$ is empty). There, we provide detailed bounds on a "large field - small field" expansion for which the above approximation is the leading term. In Remark II.1.a, below, we illustrate the sources of the smallness for $n=0$.

By Stokes' Theorem (Lemma A.1 with $r=r\left(\varepsilon_{n}\right), \sigma=\sigma_{*}=0$ and $\left.\rho=\left(\phi_{*}^{\text {crit }}\right)^{*}-\phi^{\text {crit }}\right)$ one can write

$$
\mathcal{Z}_{n}^{2|X|} e^{\left\langle\alpha^{*}, j\left(\varepsilon_{n+1}\right) \beta\right\rangle}\left[\prod_{\mathbf{x} \in X} \int_{D(\mathbf{x})} \frac{d z_{*}(\mathbf{x}) \wedge d z(\mathbf{x})}{2 \pi \imath} e^{-z_{*}(\mathbf{x}) z(\mathbf{x})}\right] e^{\tilde{\mathcal{A}}\left(\alpha^{*}, \beta ; z_{*}, z\right)}
$$

as the sum of

$$
\mathcal{Z}_{n}^{2|X|} e^{\left\langle\alpha^{*}, j\left(\varepsilon_{n+1}\right) \beta\right\rangle}\left[\prod_{\mathbf{x} \in X} \int_{|z(\mathbf{x})| \leq r\left(\varepsilon_{n}\right)} \frac{d z^{*}(\mathbf{x}) \wedge d z(\mathbf{x})}{2 \pi \imath} e^{-|z(\mathbf{x})|^{2}}\right] e^{\tilde{\mathcal{A}}\left(\alpha^{*}, \beta ; z^{*}, z\right)}
$$

and $\mathcal{Z}_{n}^{2|X|} e^{\left\langle\alpha^{*}, j\left(\varepsilon_{n+1}\right) \beta\right\rangle}$ times

$$
\begin{aligned}
& \sum_{\substack{R \subset X \\
R \neq \emptyset}} \prod_{\mathbf{x} \in R}\left[\int_{C(\mathbf{x})} \frac{d z_{*}(\mathbf{x}) \wedge d z(\mathbf{x})}{2 \pi i} e^{-z_{*}(\mathbf{x}) z(\mathbf{x})}\right] \\
& \left.\prod_{\mathbf{x} \in X \backslash R}\left[\int_{|z(\mathbf{x})| \leq r\left(\varepsilon_{n}\right)} \frac{d z(\mathbf{x})^{*} \wedge d z(\mathbf{x})}{2 \pi i} e^{-z_{*}(\mathbf{x}) z(\mathbf{x})}\right] e^{\tilde{\mathcal{A}}\left(\alpha^{*}, \beta ; z^{*}, z\right)}\right|_{\substack{\begin{subarray}{c}{z_{*}(\mathbf{x})=z(\mathbf{x})^{*} \\
\text { for } \mathbf{x} \in \mathrm{X} \backslash \mathrm{R}} }} \\
{\mid}\end{subarray}}
\end{aligned}
$$

where, for each $\mathbf{x} \in X, C(\mathbf{x})$ is a two real dimensional submanifold of $\mathbb{C}^{2}$ whose boundary is the union of "circles" $\partial D(\mathbf{x})$ and $\left\{\left(z_{*}(\mathbf{x}), z(\mathbf{x})\right) \in \mathbb{C}^{2}\left|z_{*}^{*}(\mathbf{x})=z(\mathbf{x}),\right| z(\mathbf{x}) \mid=r\left(\varepsilon_{n}\right)\right\}$. In [4] we argue that $-z_{*}(\mathbf{x}) z(\mathbf{x})$ has an extremely large negative real part whenever $\left(z_{*}(\mathbf{x}), z(\mathbf{x})\right) \in C(\mathbf{x})$. (Also see Remark II.1.b, below.) The second step in the stationary phase approximation is to ignore these terms. That is, to replace (II.4) with (II.5).

Thus, the stationary phase approximation for

$$
\int d \tilde{\mu}_{\mathrm{R}_{\varepsilon}}\left(\phi^{*}, \phi\right) I_{n}^{(\mathrm{SP})}\left(\varepsilon ; \alpha^{*}, \phi\right) I_{n}^{(\mathrm{SP})}\left(\varepsilon ; \phi^{*}, \beta\right)
$$


is

$$
(\mathrm{II} .5)=\mathcal{Z}_{n}^{2|X|} e^{\left\langle\alpha^{*}, j\left(\varepsilon_{n+1}\right) \beta\right\rangle} \int d \tilde{\mu}_{r\left(\varepsilon_{n}\right)}\left(z^{*}, z\right) e^{\tilde{\mathcal{A}}\left(\alpha^{*}, \beta ; z^{*}, z\right)}
$$

By construction

$$
\begin{aligned}
\mathcal{V}_{\varepsilon_{n}}\left(\varepsilon ; \alpha^{*}, \phi^{\text {crit }}\right)+\mathcal{V}_{\varepsilon_{n}}\left(\varepsilon ; \phi_{*}^{\text {crit }}, \beta\right) & =\mathcal{V}_{\varepsilon_{n}}\left(\varepsilon ; \alpha^{*}, j\left(\varepsilon_{n}\right) \beta\right)+\mathcal{V}_{\varepsilon_{n}}\left(\varepsilon ; j\left(\varepsilon_{n}\right) \alpha^{*}, \beta\right) \\
& =\mathcal{V}_{\varepsilon_{n+1}}\left(\varepsilon ; \alpha^{*}, \beta\right)
\end{aligned}
$$

so that

$$
\begin{aligned}
\mathcal{V}_{\varepsilon_{n}}\left(\varepsilon ; \alpha^{*}, \phi^{\text {crit }}+z\right) & +\mathcal{V}_{\varepsilon_{n}}\left(\varepsilon ; \phi_{*}^{\text {crit }}+z^{*}, \beta\right) \\
= & \mathcal{V}_{\varepsilon_{n+1}}\left(\varepsilon ; \alpha^{*}, \beta\right)+\left[\mathcal{V}_{\varepsilon_{n}}\left(\varepsilon ; \alpha^{*}, j\left(\varepsilon_{n}\right) \beta+z\right)-\mathcal{V}_{\varepsilon_{n}}\left(\varepsilon ; \alpha^{*}, j\left(\varepsilon_{n}\right) \beta\right)\right] \\
& +\left[\mathcal{V}_{\varepsilon_{n}}\left(\varepsilon ; j\left(\varepsilon_{n}\right) \alpha^{*}+z^{*}, \beta\right)-\mathcal{V}_{\varepsilon_{n}}\left(\varepsilon ; j\left(\varepsilon_{n}\right) \alpha^{*}, \beta\right)\right]
\end{aligned}
$$

Consequently, the stationary phase approximation for

$$
\int d \tilde{\mu}_{\mathrm{R}_{\varepsilon}}\left(\phi^{*}, \phi\right) I_{n}^{(\mathrm{SP})}\left(\varepsilon ; \alpha^{*}, \phi\right) I_{n}^{(\mathrm{SP})}\left(\varepsilon ; \phi^{*}, \beta\right)
$$

can also be written as

$$
\begin{aligned}
& \mathcal{Z}_{n}^{2|X|} e^{\left\langle\alpha^{*}, j\left(\varepsilon_{n+1}\right) \beta\right\rangle+\mathcal{V}_{\varepsilon_{n+1}}\left(\varepsilon ; \alpha^{*}, \beta\right)} \\
& e^{\mathcal{E}_{\varepsilon_{n}}\left(\varepsilon ; \alpha^{*}, j\left(\varepsilon_{n}\right) \beta\right)+\mathcal{E}_{\varepsilon_{n}}\left(\varepsilon ; j\left(\varepsilon_{n}\right) \alpha^{*}, \beta\right)} \int d \tilde{\mu}_{r\left(\varepsilon_{n}\right)}\left(z^{*}, z\right) e^{\partial \mathcal{A}_{\varepsilon_{n}}\left(\varepsilon ; \alpha^{*}, \beta ; z_{*}, z\right)}
\end{aligned}
$$

This is compatible with (I.7) and (I.8) since, by the definition of Appendix C,

$$
\mathcal{Z}_{2 \varepsilon_{n}}\left(\varepsilon_{n}\right)=\mathcal{Z}_{\varepsilon_{n}}(\varepsilon)^{2} \int_{|z|<r\left(\varepsilon_{n}\right)} \frac{d z^{*} \wedge d z}{2 \pi i} e^{-|z|^{2}}
$$

\section{Remark II.1}

(a) We now illustrate why the error introduced by the stationary phase approximation is extremely small, by considering the case $n=0$. The initial functional integral representation (I.1) may be written

$$
\begin{array}{r}
\operatorname{Tr} e^{-\frac{1}{k T}(H-\mu N)}=\lim _{\varepsilon \rightarrow 0} \int \prod_{\tau \in \varepsilon \mathbb{Z} \cap\left(0, \frac{1}{k T}\right]}\left\{\left[\prod_{\mathbf{x} \in X} \frac{d \alpha_{\tau}^{*}(\mathbf{x}) \wedge d \alpha_{\tau}(\mathbf{x})}{2 \pi \imath} \chi\left(\left|\alpha_{\tau}(\mathbf{x})\right|<\mathrm{R}_{\varepsilon}\right)\right] \zeta_{\varepsilon}\left(\alpha_{\tau-\varepsilon}, \alpha_{\tau}\right)\right. \\
\left.e^{-\frac{1}{2}\left\langle\alpha_{\tau-\varepsilon}^{*}, \alpha_{\tau-\varepsilon}\right\rangle} I_{0}\left(\varepsilon ; \alpha_{\tau-\varepsilon}^{*}, \alpha_{\tau}\right) e^{-\frac{1}{2}\left\langle\alpha_{\tau}^{*}, \alpha_{\tau}\right\rangle}\right\}
\end{array}
$$

where

$$
I_{0}\left(\varepsilon ; \alpha^{*}, \beta\right)=e^{\left\langle\alpha^{*}, j(\varepsilon) \beta\right\rangle} e^{-\varepsilon\left\langle\alpha^{*} \beta, v \alpha^{*} \beta\right\rangle}
$$


Dropping one of the "time derivative small field characteristic functions" $\zeta_{\varepsilon}\left(\alpha_{\tau-\varepsilon}, \alpha_{\tau}\right)$ would introduce only a very small error. This is because, writing $\alpha_{\tau-\varepsilon}=\alpha$ and $\alpha_{\tau}=\beta$, the quadratic part of the exponent of $e^{-\frac{1}{2}\left\langle\alpha^{*}, \alpha\right\rangle} I_{0}\left(\varepsilon ; \alpha^{*}, \beta\right) e^{-\frac{1}{2}\left\langle\beta^{*}, \beta\right\rangle}$ obeys

$$
\begin{aligned}
\operatorname{Re}\left\{-\frac{1}{2}\left\langle\alpha^{*}, \alpha\right\rangle+\left\langle\alpha^{*}, j(\varepsilon) \beta\right\rangle-\frac{1}{2}\left\langle\beta^{*}, \beta\right\rangle\right\} & \approx \operatorname{Re}\left\{-\frac{1}{2}\left\langle\alpha^{*}, \alpha\right\rangle+\left\langle\alpha^{*}, \beta\right\rangle-\frac{1}{2}\left\langle\beta^{*}, \beta\right\rangle\right\} \\
& =-\frac{1}{2}\|\alpha-\beta\|_{L^{2}}^{2}
\end{aligned}
$$

which generates a factor of order $e^{-\frac{1}{2} p_{0}(\varepsilon)^{2}}=e^{-\frac{1}{2} r(\varepsilon)^{2}}$ when $(\alpha, \beta)$ is not in the support of $\zeta_{\varepsilon}(\alpha, \beta)$. The quartic part $-\varepsilon\left\langle\alpha^{*} \beta, v \alpha^{*} \beta\right\rangle$ of the exponent is roughly $-\varepsilon\left\langle\alpha^{*} \alpha, v \alpha^{*} \alpha\right\rangle \leq 0$ and so cannot generate a large factor. A similar mechanism generates small factors for the right hand side of (I.3) whenever the difference $\alpha_{\tau-\varepsilon_{n}}-\alpha_{\tau}$ between the two arguments of $I_{n}$ is larger than roughly $r\left(\varepsilon_{n}\right)$.

Consequently, we apply the stationary phase approximation to the integral

$$
I_{1}\left(\varepsilon ; \alpha^{*}, \beta\right)=\int d \tilde{\mu}_{\mathrm{R}_{\varepsilon}}\left(\phi^{*}, \phi\right) \zeta_{\varepsilon}(\alpha, \phi) I_{0}\left(\varepsilon ; \alpha^{*}, \phi\right) I_{0}\left(\varepsilon ; \phi^{*}, \beta\right) \zeta_{\varepsilon}\left(\left(\phi^{*}\right)^{*}, \beta\right)
$$

of (I.2), only when the "time derivative small field condition" $\|\alpha-\beta\|_{\infty} \leq r(2 \varepsilon)$ is satisfied. The change of variables (II.2) expresses $I_{1}$ as

$$
\begin{array}{r}
I_{1}\left(\varepsilon ; \alpha^{*}, \beta\right)=e^{\left\langle\alpha^{*}, j(2 \varepsilon) \beta\right\rangle}\left[\prod_{\mathbf{x} \in X} \int_{M(\mathbf{x})} \frac{d z_{*}(\mathbf{x}) \wedge d z(\mathbf{x})}{2 \pi \imath} e^{-z_{*}(\mathbf{x}) z(\mathbf{x})}\right] e^{\tilde{\mathcal{A}}\left(\alpha^{*}, \beta ; z_{*}, z\right)} \\
\zeta_{\varepsilon}(\alpha, j(\varepsilon) \beta+z) \zeta_{\varepsilon}\left(\left(j(\varepsilon) \alpha^{*}+z_{*}\right)^{*}, \beta\right)
\end{array}
$$

The characteristic function $\zeta_{\varepsilon}(\alpha, j(\varepsilon) \beta+z)$ limits the domain of integration to $z$ 's obeying

$$
\|z+j(\varepsilon) \beta-\alpha\|_{\infty}<r(\varepsilon)
$$

Since $\|\alpha-\beta\|_{\infty} \leq r(2 \varepsilon) \leq \frac{1}{2} r(\varepsilon)$ and $\|j(\varepsilon) \beta-\beta\|_{\infty} \leq$ const $\varepsilon \mathrm{R}_{\varepsilon} \ll r(\varepsilon)$, this condition is roughly equivalent to $\|z\|_{\infty}<r(\varepsilon)$. On the difference between these two domains of integration, the integrand is extremely small, for reasons like those given above. Similarly, the condition imposed by the second $\zeta_{\varepsilon}$ is roughly equivalent to $\left\|z_{*}\right\|_{\infty}<r(\varepsilon)$. The two conditions $\|z\|_{\infty} \leq r(\varepsilon)$ and $\left\|z_{*}\right\|_{\infty} \leq r(\varepsilon)$ are built into the domains of integration $D(\mathbf{x})$ in (II.4).

(b) The "time derivative small field condition" $\|\alpha-\beta\|_{\infty} \leq r(2 \varepsilon) \leq \frac{1}{2} r(\varepsilon)$ is also used to ensure that $-z_{*}(\mathbf{x}) z(\mathbf{x})$ has an extremely large negative real part whenever $\left(z_{*}(\mathbf{x}), z(\mathbf{x})\right)$ lies on $C(\mathbf{x})$, the side of the Stokes' "cylinder". This may be seen from Remark A.3 with $r=r(\varepsilon), \sigma=\sigma_{*}=0$ and $\rho=\left(\phi_{*}^{\text {crit }}\right)^{*}-\phi^{\text {crit }}=j(\varepsilon)[\alpha-\beta]$. 


\section{The Induction Step}

In preparation for the proof of the Proposition III.3 below, as well as of the proofs of Theorems I.3 and I.4, we collect consequences of the hypotheses I.1 in the form in which they are actually used in these proofs.

Remark III.1 Hypothesis I.1 implies that for all $0 \leq \delta \leq \Theta / 2$

$$
\begin{aligned}
K_{E} & \geq 2^{19} e^{9 K_{j}} \\
{\left[\frac{2^{31} e^{14 \delta K_{j}}}{K_{E}}+e^{2 \delta K_{j}}\left(\frac{\kappa(\delta)}{\kappa(2 \delta)}\right)^{2}\right]\left(\frac{r(\delta)}{r(2 \delta)}\right)^{2} } & \leq 2 \\
2 e^{2 \delta K_{j}}\left(\frac{\kappa(2 \delta)}{\kappa(\delta)}\right)^{4}+2^{26} e^{9 K_{j}} \delta\|v\| \| r(\delta) \kappa(2 \delta)^{3} & \leq q
\end{aligned}
$$

Proof: Hypothesis I.1.v forces $C\left(\Theta, K_{E}\right) \geq 0$ and hence $K_{E} \geq 2^{33} e^{14 K_{j}}$, which implies (III.1.i). Hypothesis I.1.v also forces

$$
\left(\frac{\kappa(\delta)}{\kappa(2 \delta)}\right)^{2} \leq 2 e^{-2 \Theta K_{j}} \sqrt{1-\frac{2^{33} e^{14 K_{j}}}{K_{E}}}\left(\frac{r(2 \delta)}{r(\delta)}\right)^{2} \leq 2 e^{-2 \delta K_{j}}\left[1-\frac{2^{32} e^{14 K_{j}}}{K_{E}}\right]\left(\frac{r(2 \delta)}{r(\delta)}\right)^{2}
$$

and hence

$$
4 \frac{2^{31} e^{14 \delta K_{j}}}{K_{E}}+e^{2 \delta K_{j}}\left(\frac{\kappa(\delta)}{\kappa(2 \delta)}\right)^{2}\left(\frac{r(\delta)}{r(2 \delta)}\right)^{2} \leq 2
$$

So (III.1.ii) now follows from Hypothesis I.1.i, which ensures that $\left(\frac{r(\delta)}{r(2 \delta)}\right)^{2} \leq 4$. Finally, by Hypotheses I.1.iv and I.1.v,

$$
\begin{aligned}
2 e^{2 \delta K_{j}\left(\frac{\kappa(2 \delta)}{\kappa(\delta)}\right)^{4}+2^{26} e^{9 K_{j}} \delta\|v\| r(\delta) \kappa(2 \delta)^{3}} & \leq e^{2 \Theta K_{j}} q C\left(\Theta, K_{E}\right)+\frac{2^{26} e^{9 K_{j}}}{K_{E}} \\
& =q e^{-2 \Theta K_{j}}-q \frac{2^{33} e^{-2 \Theta K_{j}} e^{14 K_{j}}}{K_{E}}+\frac{2^{26} e^{9 K_{j}}}{K_{E}} \\
& \leq q-\frac{2^{33} e^{12 K_{j}}}{K_{E}}+\frac{2^{26} e^{9 K_{j}}}{K_{E}} \\
& \leq q
\end{aligned}
$$

since $q \geq 1$ and $0<\Theta \leq 1$.

We formulate the recursion relation (I.8) that defines $\mathcal{E}_{\varepsilon_{n}}\left(\varepsilon ; \alpha^{*}, \beta\right)$ more abstractly.

Definition III.2 Let $0 \leq \varepsilon \leq \delta$. For an action $\mathcal{E}\left(\alpha^{*}, \beta\right)$ we set

$$
\mathfrak{R}_{\delta, \varepsilon}[\mathcal{E}]\left(\alpha^{*}, \beta\right)=\mathcal{E}\left(\alpha^{*}, j(\delta) \beta\right)+\mathcal{E}\left(j(\delta) \alpha^{*}, \beta\right)+\log \frac{\int d \tilde{\mu}_{r(\delta)}\left(z^{*}, z\right) e^{\partial \mathcal{A}_{\delta, \varepsilon}\left(\mathcal{E} ; \alpha^{*}, \beta ; z^{*}, z\right)}}{\int d \tilde{\mu}_{r(\delta)}\left(z^{*}, z\right)}
$$


whenever the logarithm is defined. Here

$$
\begin{aligned}
\partial \mathcal{A}_{\delta, \varepsilon}\left(\mathcal{E} ; \alpha^{*}, \beta ; z_{*}, z\right)= & {\left[\mathcal{V}_{\delta}\left(\varepsilon ; \alpha^{*}, j(\delta) \beta+z\right)-\mathcal{V}_{\delta}\left(\varepsilon ; \alpha^{*}, j(\delta) \beta\right)\right] } \\
& +\left[\mathcal{V}_{\delta}\left(\varepsilon ; j(\delta) \alpha^{*}+z_{*}, \beta\right)-\mathcal{V}_{\delta}\left(\varepsilon ; j(\delta) \alpha^{*}, \beta\right)\right] \\
& +\left[\mathcal{E}\left(\alpha^{*}, j(\delta) \beta+z\right)-\mathcal{E}\left(\alpha^{*}, j(\delta) \beta\right)\right] \\
& +\left[\mathcal{E}\left(j(\delta) \alpha^{*}+z_{*}, \beta\right)-\mathcal{E}\left(j(\delta) \alpha^{*}, \beta\right)\right]
\end{aligned}
$$

The recursion relation (I.8) is equivalent to

$$
\begin{aligned}
\mathcal{E}_{\varepsilon}\left(\varepsilon ; \alpha^{*}, \beta\right) & =0 \\
\mathcal{E}_{\varepsilon_{n+1}}\left(\varepsilon ; \alpha^{*}, \beta\right) & =\mathfrak{R}_{\varepsilon_{n}, \varepsilon}\left[\mathcal{E}_{\varepsilon_{n}}\left(\varepsilon ; \alpha^{*}, \beta\right)\right]
\end{aligned}
$$

To prove Theorem I.3, we perform induction on $n$ to successively bound $\mathcal{E}_{\varepsilon_{n}}(\varepsilon ; \cdot)$ for $n=0, \cdots, \log _{2} \frac{\Theta}{\varepsilon}$. For the induction step, we use

Proposition III.3 Assume the $r(t)$ and $\kappa(t)$ fulfill the Hypotheses I.1. Then, for all $0 \leq \varepsilon \leq \delta \leq \Theta / 2$, with $\delta$ an integer multiple of $\varepsilon$, the following holds:

Let $\mathcal{E}\left(\alpha^{*}, \beta\right)$ be an analytic function which has degree at least two both in $\alpha^{*}$ and $\beta$ and which obeys $\left\|\mathcal{E}\left(\alpha^{*}, \beta\right)\right\|_{\delta} \leq 2^{9} e^{7 \delta K_{j}} \delta\|v \mid\| r(\delta) \kappa(2 \delta)^{3}$. Then $\mathfrak{R}_{\delta, \varepsilon}[\mathcal{E}]\left(\alpha^{*}, \beta\right)$ is well defined, has degree at least two both in $\alpha^{*}$ and $\beta$, and satisfies the estimate

$$
\left\|\Re_{\delta, \varepsilon}[\mathcal{E}]\right\|_{2 \delta} \leq 2^{32} e^{14 \delta K_{j}} \delta^{2}\|v\|^{2} r(\delta)^{2} \kappa(2 \delta)^{6}+2 e^{2 \delta K_{j}}\left(\frac{\kappa(2 \delta)}{\kappa(\delta)}\right)^{4}\|\mathcal{E}\|_{\delta}
$$

Proof: Observe that the functions $\mathcal{V}_{\delta}\left(\varepsilon ; \alpha^{*}, j(\delta) \beta+z\right)-\mathcal{V}_{\delta}\left(\varepsilon ; \alpha^{*}, j(\delta) \beta\right)$ and $\mathcal{E}\left(\alpha^{*}, j(\delta) \beta+z\right)-\mathcal{E}\left(\alpha^{*}, j(\delta) \beta\right)$ have both degree at least two in $\alpha^{*}$, degree at least one in $z$ and do not depend on $z_{*}$. Similarly, both $\mathcal{V}_{\delta}\left(\varepsilon ; j(\delta) \alpha^{*}+z_{*}, \beta\right)-\mathcal{V}_{\delta}\left(\varepsilon ; j(\delta) \alpha^{*}, \beta\right)$ and $\mathcal{E}\left(j(\delta) \alpha^{*}+z_{*}, \beta\right)-\mathcal{E}\left(j(\delta) \alpha^{*}, \beta\right)$ have degree at least two in $\beta$, degree at least one in $z_{*}$ and do not depend on $z$. Since the integral of any monomial against $d \tilde{\mu}_{r(\delta)}\left(z^{*}, z\right)$ is zero unless there are the same number of $z$ 's and $z^{*}$ 's,

$$
\int d \tilde{\mu}_{r(\delta)}\left(z^{*}, z\right) \partial \mathcal{A}_{\delta, \varepsilon}\left(\mathcal{E} ; \alpha^{*}, \beta ; z^{*}, z\right)=0
$$

and $\log \frac{\int d \tilde{\mu}_{r(\delta)}\left(z^{*}, z\right) e^{\partial \mathcal{A}_{\delta, \varepsilon}\left(\mathcal{E} ; \alpha^{*}, \beta ; z^{*}, z\right)}}{\int d \tilde{\mu}_{r(\delta)}\left(z^{*}, z\right)}$ has degree at least two both in $\alpha^{*}$ and $\beta$. This implies that $\mathfrak{R}_{\delta, \varepsilon}[\mathcal{E}]\left(\alpha^{*}, \beta\right)$ has degree at least two both in $\alpha^{*}$ and $\beta$.

To estimate $\partial A_{\delta, \varepsilon}$ we introduce a second auxiliary weight system $w_{\text {fluct }}$. It has metric $\mathrm{m} d$ and associates the constant weight factor $\kappa(2 \delta)$ to the fields $\alpha^{*}$ and $\beta$, and 
the constant weight factor $4 r(\delta)$ to the fluctuation fields $z_{*}$ and $z$. We abbreviate $\left\|f\left(\alpha^{*}, \beta ; z_{*}, z\right)\right\|_{\text {fluct }}=\left\|f\left(\alpha^{*}, \beta ; z_{*}, z\right)\right\|_{w_{\text {fluct }}}$. Clearly, $\left\|f\left(\alpha^{*}, \beta\right)\right\|_{2 \delta}=\left\|f\left(\alpha^{*}, \beta\right)\right\|_{\text {fluct }}$ for functions that are independent of the fluctuation fields.

Observe that

$$
\begin{aligned}
\mathcal{V}_{\delta}\left(\varepsilon ; \alpha^{*}, j(\delta) \beta+z\right)-\mathcal{V}_{\delta} & \left(\varepsilon ; \alpha^{*}, j(\delta) \beta\right) \\
= & \varepsilon \sum_{\tau \in \varepsilon \mathbb{Z} \cap(0, \delta]}\left[\left\langle\gamma_{* \tau-\varepsilon} g_{\tau}, v \gamma_{* \tau-\varepsilon} g_{\tau}\right\rangle-\left\langle\gamma_{* \tau-\varepsilon} \hat{g}_{\tau}, v \gamma_{* \tau-\varepsilon} \hat{g}_{\tau}\right\rangle\right]
\end{aligned}
$$

with

$$
\gamma_{* \tau}=j(\tau) \alpha^{*} \quad g_{\tau}=j(2 \delta-\tau) \beta \quad \hat{g}_{\tau}=j(\delta-\tau)(j(\delta) \beta+z)=j(2 \delta-\tau) \beta+j(\delta-\tau) z
$$

We apply [3, Proposition A.3.ii], with $d$ replaced by $\mathrm{m} d, r=4, s=3, h\left(\gamma_{1}, \cdots, \gamma_{4}\right)=$ $\left\langle\gamma_{1} \gamma_{2}, v \gamma_{3} \gamma_{4}\right\rangle, \alpha_{1}=\alpha^{*}, \alpha_{2}=\beta, \alpha_{3}=z$, weights $\lambda_{1}=\cdots=\lambda_{4}=1$ and

$$
\begin{array}{lll}
\Gamma_{1}^{1}=\Gamma_{3}^{1}=j(\tau-\varepsilon) & \Gamma_{2}^{2}=\Gamma_{4}^{2}=j(2 \delta-\tau) & \Gamma_{2}^{3}=\Gamma_{4}^{3}=0 \\
\tilde{\Gamma}_{1}^{1}=\tilde{\Gamma}_{3}^{1}=j(\tau-\varepsilon) & \tilde{\Gamma}_{2}^{2}=\tilde{\Gamma}_{4}^{2}=j(2 \delta-\tau) & \tilde{\Gamma}_{2}^{3}=\tilde{\Gamma}_{4}^{3}=j(\delta-\tau)
\end{array}
$$

with all other $\Gamma_{i}^{j}$ s and $\tilde{\Gamma}_{i}^{j}$ s s being zero. Then

$$
\begin{aligned}
\sigma & =\kappa(2 \delta) \max \left\{\|j(\tau-\varepsilon)\|, \quad\|j(2 \delta-\tau)\|\|, \quad\| j(2 \delta-\tau)\left\|+4 \frac{r(\delta)}{\kappa(2 \delta)}\right\| j(\delta-\tau) \|\right\} \\
& \leq\left(1+4 \frac{r(\delta)}{\kappa(2 \delta)}\right) e^{2 K_{j} \delta} \kappa(2 \delta) \\
\sigma_{\delta} & =4 r(\delta)\|j(\delta-\tau)\| \leq 4 e^{K_{j} \delta} r(\delta)
\end{aligned}
$$

So, for each $\tau \in \varepsilon \mathbb{Z} \cap(0, \delta]$,

$$
\begin{aligned}
\left\|\left\langle\gamma_{* \tau-\varepsilon} g_{\tau}, v \gamma_{* \tau-\varepsilon} g_{\tau}\right\rangle-\left\langle\gamma_{* \tau-\varepsilon} \hat{g}_{\tau}, v \gamma_{* \tau-\varepsilon} \hat{g}_{\tau}\right\rangle\right\|_{\text {fluct }} & \leq 4\|h\|_{w_{\lambda}} \sigma_{\delta} \sigma^{3} \\
& \leq 2^{9} e^{7 K_{j} \delta}\|v\| r(\delta) \kappa(2 \delta)^{3}
\end{aligned}
$$

Here, we used that $r(\delta) \leq \frac{1}{4} \kappa(\delta) \leq \frac{1}{2} \kappa(2 \delta)$ by Hypotheses I.1.ii and I.1.i. Summing over $\tau$ gives

$$
\left\|\mathcal{V}_{\delta}\left(\varepsilon ; \alpha^{*}, j(\delta) \beta+z\right)-\mathcal{V}_{\delta}\left(\varepsilon ; \alpha^{*}, j(\delta) \beta\right)\right\|_{\text {fluct }} \leq 2^{9} e^{7 K_{j} \delta} \delta\|v\| \| r(\delta) \kappa(2 \delta)^{3}
$$

Similarly

$$
\left\|\mathcal{V}_{\delta}\left(\varepsilon ; j(\delta) \alpha^{*}+z_{*}, \beta\right)-\mathcal{V}_{\delta}\left(\varepsilon ; j(\delta) \alpha^{*}+z_{*}, \beta\right)\right\|_{\text {fluct }} \leq 2^{9} e^{7 K_{j} \delta} \delta\|v\| \| r(\delta) \kappa(2 \delta)^{3}
$$

Next, by [3, Corollary A.2], for any analytic function $f\left(\alpha^{*}, \beta\right)$

$$
\left\|f\left(\alpha^{*}, j(\delta) \beta+z\right)-f\left(\alpha^{*}, j(\delta) \beta\right)\right\|_{\text {fluct }} \leq\left\|f\left(\alpha^{*}, j(\delta) \beta+z\right)\right\|_{\text {fluct }} \leq\left\|f\left(\alpha^{*}, \beta\right)\right\|_{\delta}
$$


since

$$
\frac{\kappa(2 \delta)}{\kappa(\delta)}\|\| j(\delta)\left\|\mid+\frac{4 r(\delta)}{\kappa(\delta)}\right\| 1\|\| \leq e^{\delta K_{j}} \frac{\kappa(2 \delta)}{\kappa(\delta)}+\frac{4 r(\delta)}{\kappa(\delta)} \leq 1
$$

by Hypothesis I.1.ii. In particular $\left\|\mathcal{E}\left(\alpha^{*}, j(\delta) \beta+z\right)-\mathcal{E}\left(\alpha^{*}, j(\delta) \beta\right)\right\|_{\text {fluct }} \leq\|\mathcal{E}\|_{\delta}$. Similarly $\left\|\mathcal{E}\left(j(\delta) \alpha^{*}+z_{*}, \beta\right)-\mathcal{E}\left(j(\delta) \alpha^{*}, \beta\right)\right\|_{\text {fluct }} \leq\|\mathcal{E}\|_{\delta}$.

Combining the bounds of the previous two paragraphs with the assumption on $\|\mathcal{E}\|_{\delta}$, we get

$$
\left\|\partial A_{\delta, \varepsilon}(\mathcal{E} ; \cdot)\right\|_{\text {fluct }} \leq 2^{10} e^{7 \delta K_{j}} \delta\|v\| r(\delta) \kappa(2 \delta)^{3}+2\|\mathcal{E}\|_{\delta} \leq 2^{11} e^{7 \delta K_{j}} \delta\|v\| r(\delta) \kappa(2 \delta)^{3} \leq \frac{1}{64}
$$

by Hypothesis I.1.iv and (III.1.i). By (III.3) and [3, Corollary 3.5] with $n=1$

$$
\begin{aligned}
\left\|\log \frac{\int d \tilde{\mu}_{r(\delta)}\left(z^{*}, z\right) e^{\partial \mathcal{A}_{\delta, \varepsilon}\left(\mathcal{E} ; \alpha^{*}, \beta ; z_{*}, z\right)}}{\int d \tilde{\mu}_{r(\delta)}\left(z^{*}, z\right)}\right\|_{2 \delta} & \leq \frac{\left\|\partial A_{\delta, \varepsilon}(\mathcal{E} ; \cdot)\right\|_{\text {fluct }}^{2}}{\left(\frac{1}{20}-\left\|\partial A_{\delta, \varepsilon}(\mathcal{E} ; \cdot)\right\|_{\text {fluct }}\right)^{2}} \\
& \leq 2^{32} e^{14 \delta K_{j}} \delta^{2}\|v\| \|^{2} r(\delta)^{2} \kappa(2 \delta)^{6}
\end{aligned}
$$

Combining this estimate and the estimate of Lemma III.4, below, with $f=\mathcal{E}$, we get the desired bound on $\left\|\Re_{\delta, \varepsilon}[\mathcal{E}]\right\|_{2 \delta}$.

Lemma III.4 Let $f\left(\alpha^{*}, \beta\right)$ be an analytic function that has degree at least two both in $\alpha^{*}$ and $\beta$. Then

$$
\left\|f\left(\alpha^{*}, j(\delta) \beta\right)\right\|_{2 \delta},\left\|f\left(j(\delta) \alpha^{*}, \beta\right)\right\|_{2 \delta} \leq e^{2 \delta K_{j}}\left(\frac{\kappa(2 \delta)}{\kappa(\delta)}\right)^{4}\|f\|_{\delta}
$$

Proof: Introduce the auxiliary weight system $w_{\text {aux }}$ with, in the language of [3, Definitions 2.5 and 2.6 and, more specifically, (A.1)], metric $\mathrm{m} d$ that associates the constant weight factor $\kappa(\delta)$ to the field $\alpha_{*}$ and the constant weight factor $e^{-\delta K_{j}} \kappa(\delta)$ to the field $\beta$. Since, by (I.11), $\frac{e^{-\delta K_{j}} \kappa(\delta)}{\kappa(\delta)}\|j(\delta)\| \leq 1$, [3, Corollary A.2] gives

$$
\left\|f\left(\alpha^{*}, j(\delta) \beta\right)\right\|_{w_{\text {aux }}} \leq\|f\|_{\delta}
$$

As $f\left(\alpha^{*}, j(\delta) \beta\right)$ has degree at least two both in $\alpha^{*}$ and $\beta$ and $e^{-\delta K_{j}} \kappa(\delta) \geq \kappa(2 \delta)$, by Hypothesis I.1.ii,

$$
\left\|f\left(\alpha^{*}, j(\delta) \beta\right)\right\|_{2 \delta} \leq\left(\frac{\kappa(2 \delta)}{\kappa(\delta)}\right)^{2}\left(\frac{\kappa(2 \delta)}{e^{-\delta K_{j}} \kappa(\delta)}\right)^{2}\left\|f\left(\alpha^{*}, j(\delta) \beta\right)\right\|_{w_{\text {aux }}} \leq e^{2 \delta K_{j}}\left(\frac{\kappa(2 \delta)}{\kappa(\delta)}\right)^{4}\|f\|_{\delta}
$$

The estimate on $\left\|f\left(j(\delta) \alpha^{*}, \beta\right)\right\|_{2 \delta}$ is similar. 
Proof of Theorem I.3: We write $\delta=\varepsilon_{n}=2^{n} \varepsilon$ and prove the statement by induction on $n$. In the case $n=0$ there is nothing to prove. For the induction step from $n$ to $n+1$, set $\delta=\varepsilon_{n}$. The hypothesis of Proposition III.3, with $\mathcal{E}=\mathcal{E}_{\delta}$, is satisfied since,

$$
\left\|\mathcal{E}_{\delta}\right\|_{\delta} \leq K_{E} \delta^{2}\|v\|^{2} r(\delta)^{2} \kappa(\delta)^{6} \leq 8 \delta\|\| v\|\| r(\delta) \kappa(2 \delta)^{3}
$$

by the inductive hypothesis and Hypothesis I.1, parts (i) and (iv). Using (III.2), Proposition III.3 and (III.1.ii) we see that

$$
\begin{aligned}
\left\|\mathcal{E}_{\varepsilon_{n+1}}\right\|_{\varepsilon_{n+1}} & \leq 2^{32} e^{14 \delta K_{j}} \delta^{2}\|v\|^{2} r(\delta)^{2} \kappa(2 \delta)^{6}+2 e^{2 \delta K_{j}}\left(\frac{\kappa(2 \delta)}{\kappa(\delta)}\right)^{4} K_{E} \delta^{2}\|v\|^{2} r(\delta)^{2} \kappa(\delta)^{6} \\
& \leq\left[2^{32} e^{14 \delta K_{j}}+2 e^{2 \delta K_{j}}\left(\frac{\kappa(\delta)}{\kappa(2 \delta)}\right)^{2} K_{E}\right] \delta^{2}\|v\|^{2} r(\delta)^{2} \kappa(2 \delta)^{6} \\
& =\frac{1}{2}\left[\frac{2^{31} e^{14 \delta K_{j}}}{K_{E}}+e^{2 \delta K_{j}}\left(\frac{\kappa(\delta)}{\kappa(2 \delta)}\right)^{2}\right]\left(\frac{r(\delta)}{r(2 \delta)}\right)^{2} K_{E}(2 \delta)^{2}\|v\| \|^{2} r(2 \delta)^{2} \kappa(2 \delta)^{6} \\
& \leq K_{E}(2 \delta)^{2}\|v\|^{2} r(2 \delta)^{2} \kappa(2 \delta)^{6} \\
& =K_{E} \varepsilon_{n+1}^{2}\|v\|^{2} r\left(\varepsilon_{n+1}\right)^{2} \kappa\left(\varepsilon_{n+1}\right)^{6}
\end{aligned}
$$

In the proof of Theorem I.4, we shall compare $\mathcal{E}_{\theta}\left(2^{-m-1} \theta ; \alpha^{*}, \beta\right)$ and $\mathcal{E}_{\theta}\left(2^{-m} \theta ; \alpha^{*}, \beta\right)$ to prove that the sequence $\mathcal{E}_{\theta}\left(2^{-m} \theta ;\right.$. ) is Cauchy with respect to our norm. To do so, we shall compare $\mathcal{E}_{\varepsilon_{n}}\left(\frac{\varepsilon}{2} ; \cdot\right)$ and $\mathcal{E}_{\varepsilon_{n}}(\varepsilon ; \cdot)$ for each $n=0, \cdots, \log _{2} \frac{\Theta}{\varepsilon}$. This is done by induction on $n$. For the induction step, we use Proposition III.6, below. To prepare for it, we have

Lemma III.5 Set

$$
W\left(\alpha_{*}, \beta\right)=\mathcal{V}_{\delta}\left(\varepsilon ; \alpha_{*}, \beta\right)-\mathcal{V}_{\delta}\left(\frac{\varepsilon}{2} ; \alpha_{*}, \beta\right)
$$

Then

$$
\begin{aligned}
\left\|W\left(\alpha_{*}, z+j(\delta) \beta\right)-W\left(\alpha_{*}, j(\delta) \beta\right)\right\|_{\text {fluct }} & \leq 2^{10} e^{9 K_{j}} \varepsilon \delta\|v\| r(\delta) \kappa(2 \delta)^{3} \\
\left\|W\left(z_{*}+j(\delta) \alpha_{*}, \beta\right)-W\left(j(\delta) \alpha_{*}, \beta\right)\right\|_{\text {fluct }} & \leq 2^{10} e^{9 K_{j}} \varepsilon \delta\|v\| r(\delta) \kappa(2 \delta)^{3}
\end{aligned}
$$

Proof: We prove the first inequality. By definition

$$
W\left(\alpha_{*}, \beta\right)=W_{1}\left(\alpha_{*}, \beta\right)+W_{2}\left(\alpha_{*}, \beta\right)
$$


with

$$
\begin{aligned}
& W_{1}\left(\alpha_{*}, \beta\right)=-\frac{\varepsilon}{2} \sum_{\tau \in \varepsilon \mathbb{Z} \cap(0, \delta]}\left[\left\langle\gamma_{* \tau-\varepsilon} \gamma_{\tau}, v \gamma_{* \tau-\varepsilon} \gamma_{\tau}\right\rangle-\left\langle\gamma_{* \tau-\varepsilon} \gamma_{\tau-\frac{\varepsilon}{2}}, v \gamma_{* \tau-\varepsilon} \gamma_{\tau-\frac{\varepsilon}{2}}\right\rangle\right] \\
& W_{2}\left(\alpha_{*}, \beta\right)=-\frac{\varepsilon}{2} \sum_{\tau \in \varepsilon \mathbb{Z} \cap(0, \delta]}\left[\left\langle\gamma_{* \tau-\varepsilon} \gamma_{\tau}, v \gamma_{* \tau-\varepsilon} \gamma_{\tau}\right\rangle-\left\langle\gamma_{* \tau-\frac{\varepsilon}{2}} \gamma_{\tau}, v \gamma_{* \tau-\frac{\varepsilon}{2}} \gamma_{\tau}\right\rangle\right]
\end{aligned}
$$

where

$$
\gamma_{* \tau}=j(\tau) \alpha^{*} \quad \gamma_{\tau}=j(\delta-\tau)
$$

Using the notation of (III.4),

$$
\begin{aligned}
& W_{1}\left(\alpha_{*}, j(\delta) \beta\right)- W_{1}\left(\alpha_{*}, z+j(\delta) \beta\right) \\
&=-\frac{\varepsilon}{2} \sum_{\tau \in \varepsilon \mathbb{Z} \cap(0, \delta]} {\left[\left\langle\gamma_{* \tau-\varepsilon} g_{\tau}, v \gamma_{* \tau-\varepsilon} g_{\tau}\right\rangle-\left\langle\gamma_{* \tau-\varepsilon} g_{\tau-\frac{\varepsilon}{2}}, v \gamma_{* \tau-\varepsilon} g_{\tau-\frac{\varepsilon}{2}}\right\rangle\right.} \\
&-\left.-\left\langle\gamma_{* \tau-\varepsilon} \hat{g}_{\tau}, v \gamma_{* \tau-\varepsilon} \hat{g}_{\tau}\right\rangle+\left\langle\gamma_{* \tau-\varepsilon} \hat{g}_{\tau-\frac{\varepsilon}{2}}, v \gamma_{* \tau-\varepsilon} \hat{g}_{\tau-\frac{\varepsilon}{2}}\right\rangle\right] \\
&=-\frac{\varepsilon}{2} \sum_{\tau \in \varepsilon \mathbb{Z} \cap(0, \delta]} {\left[\left\langle\gamma_{* \tau-\varepsilon} g_{\tau}, v \gamma_{* \tau-\varepsilon} g_{\tau}\right\rangle-\left\langle\gamma_{* \tau-\varepsilon} j\left(\frac{\varepsilon}{2}\right) g_{\tau}, v \gamma_{* \tau-\varepsilon} j\left(\frac{\varepsilon}{2}\right) g_{\tau}\right\rangle\right.} \\
&\left.-\left\langle\gamma_{* \tau-\varepsilon} \hat{g}_{\tau}, v \gamma_{* \tau-\varepsilon} \hat{g}_{\tau}\right\rangle+\left\langle\gamma_{* \tau-\varepsilon} j\left(\frac{\varepsilon}{2}\right) \hat{g}_{\tau}, v \gamma_{* \tau-\varepsilon} j\left(\frac{\varepsilon}{2}\right) \hat{g}_{\tau}\right\rangle\right]
\end{aligned}
$$

This time, we apply [3, Proposition A.3.iii] using the $\Gamma_{i}^{j}$ 's and $\tilde{\Gamma}_{i}^{j}$ 's of (III.5) and, in addition,

$$
A_{1}=\tilde{A}_{1}=A_{3}=\tilde{A}_{3}=\mathbb{1} \quad A_{2}=A_{4}=\mathbb{1} \quad \tilde{A}_{2}=\tilde{A}_{4}=j\left(\frac{\varepsilon}{2}\right)
$$

The Corollary bounds the $\|$ - $\|_{\text {fluct }}$ norm of the $\tau$ term by $4^{2}\|v\| \| \sigma_{\delta} a_{\delta}(\sigma a)^{3}$ with the $\sigma$ and $\sigma_{\delta}$ of (III.6) and

$$
\begin{aligned}
a & =\max \left\{\|\mathbb{1}\|\left|, \quad\left\|j\left(\frac{\varepsilon}{2}\right) \mid\right\|\right\} \leq e^{K_{j} \frac{\varepsilon}{2}}\right. \\
a_{\delta} & =\left\|j\left(\frac{\varepsilon}{2}\right)-\mathbb{1}\right\| \mid \leq \frac{\varepsilon}{2} K_{j} e^{K_{j} \frac{\varepsilon}{2}}
\end{aligned}
$$

by (I.11). Inserting and summing over $\tau$, we get

$$
\begin{aligned}
\| W_{1}\left(\alpha_{*}, z\right. & +j(\delta) \beta)-W_{1}\left(\alpha_{*}, j(\delta) \beta\right) \|_{\text {fluct }} \\
& \leq \frac{\varepsilon}{2} \frac{\delta}{\varepsilon} 4^{2}\|v\| 4 e^{K_{j} \delta} r(\delta) \frac{\varepsilon}{2} K_{j} e^{K_{j} \frac{\varepsilon}{2}}\left(\left(1+4 \frac{r(\delta)}{\kappa(2 \delta)}\right) e^{2 K_{j} \delta} \kappa(2 \delta) e^{K_{j} \frac{\varepsilon}{2}}\right)^{3} \\
& \leq 2^{9} e^{9 K_{j}} \varepsilon \delta\|v\| r(\delta) \kappa(2 \delta)^{3}
\end{aligned}
$$

The same estimate holds for $\left\|W_{2}\left(\alpha_{*}, z+j(\delta) \beta\right)-W_{2}\left(\alpha_{*}, j(\delta) \beta\right)\right\|_{\text {fluct }}$. 
Proposition III.6 Under the hypotheses of Proposition III.3, assume that there is a second analytic function $\tilde{\mathcal{E}}\left(\alpha^{*}, \beta\right)$ which has similar properties to $\mathcal{E}$ and is close to $\mathcal{E}$. Precisely, we assume that $\tilde{\mathcal{E}}$ is of degree at least two both in $\alpha^{*}$ and $\beta$ and obeys $\|\tilde{\mathcal{E}}\|_{\delta} \leq 2^{9} e^{7 \delta K_{j}} \delta\|v\| \| r(\delta) \kappa(2 \delta)^{3}$. Then

$$
\left\|\Re_{\delta, \varepsilon}[\mathcal{E}]-\mathfrak{R}_{\delta, \frac{\varepsilon}{2}}[\tilde{\mathcal{E}}]\right\|_{2 \delta} \leq 2^{36} e^{18 K_{j}} \varepsilon \delta^{2}\|v\|^{2} r(\delta)^{2} \kappa(2 \delta)^{6}+q\|\mathcal{E}-\tilde{\mathcal{E}}\|_{\delta}
$$

where $q$ is the constant in parts (v) and (vi) of Hypotheses I.1.

Proof: By Definition III.2,

$$
\begin{aligned}
\mathfrak{R}_{\delta, \varepsilon}[\mathcal{E}]-\mathfrak{R}_{\delta, \frac{\varepsilon}{2}}[\tilde{\mathcal{E}}]=\mathcal{B}\left(\alpha^{*}, \beta\right) & +\log \frac{\int d \tilde{\mu}_{r(\delta)}\left(z^{*}, z\right) e^{\partial \mathcal{A}_{\delta, \varepsilon}\left(\mathcal{E} ; \alpha^{*}, \beta ; z^{*}, z\right)}}{\int d \tilde{\mu}_{r(\delta)}\left(z^{*}, z\right)} \\
& -\log \frac{\int d \tilde{\mu}_{r(\delta)}\left(z^{*}, z\right) e^{\partial \mathcal{A}_{\delta, \frac{\varepsilon}{2}}\left(\tilde{\mathcal{E}} ; \alpha^{*}, \beta ; z^{*}, z\right)}}{\int d \tilde{\mu}_{r(\delta)}\left(z^{*}, z\right)}
\end{aligned}
$$

where $\mathcal{B}\left(\alpha^{*}, \beta\right)=(\mathcal{E}-\tilde{\mathcal{E}})\left(\alpha^{*}, j(\delta) \beta\right)+(\mathcal{E}-\tilde{\mathcal{E}})\left(j(\delta) \alpha^{*}, \beta\right)$. By Lemma III.4,

$$
\|\mathcal{B}\|_{2 \delta} \leq 2 e^{2 \delta K_{j}}\left(\frac{\kappa(2 \delta)}{\kappa(\delta)}\right)^{4}\|\mathcal{E}-\tilde{\mathcal{E}}\|_{\delta}
$$

With the notation of the previous lemma,

$$
\begin{aligned}
\partial \mathcal{A}_{\delta, \varepsilon}\left(\mathcal{E} ; \alpha^{*}, \beta ; z_{*}, z\right)-\partial \mathcal{A}_{\delta, \frac{\varepsilon}{2}}\left(\tilde{\mathcal{E}} ; \alpha^{*}, \beta ; z_{*}, z\right) \\
=\left[W\left(\alpha^{*}, z+j(\delta) \beta\right)-W\left(\alpha^{*}, j(\delta) \beta\right)\right]+\left[W\left(z_{*}+j(\delta) \alpha^{*}, \beta\right)-W\left(j(\delta) \alpha^{*}, \beta\right)\right] \\
\quad+\mathcal{C}\left(\alpha^{*}, \beta ; z_{*}, z\right)
\end{aligned}
$$

where

$$
\begin{aligned}
\mathcal{C}\left(\alpha^{*}, \beta ; z_{*}, z\right)=\left[(\mathcal{E}-\tilde{\mathcal{E}})\left(\alpha^{*}, j(\delta) \beta+z\right)-(\mathcal{E}-\tilde{\mathcal{E}})\left(\alpha^{*}, j(\delta) \beta\right)\right] \\
+ \\
+\left[(\mathcal{E}-\tilde{\mathcal{E}})\left(j(\delta) \alpha^{*}+z_{*}, \beta\right)-(\mathcal{E}-\tilde{\mathcal{E}})\left(j(\delta) \alpha^{*}, \beta\right)\right]
\end{aligned}
$$

By (III.7), $\|\mathcal{C}\|_{\text {fluct }} \leq 2\|\mathcal{E}-\tilde{\mathcal{E}}\|_{\delta}$. Combining this with Lemma III.5, we get

$$
\begin{gathered}
\left\|\partial \mathcal{A}_{\delta, \varepsilon}(\mathcal{E} ; \cdot)-\partial \mathcal{A}_{\delta, \frac{\varepsilon}{2}}(\tilde{\mathcal{E}} ; \cdot)\right\|_{\text {fluct }} \leq 2^{11} e^{9 K_{j}} \varepsilon \delta\|v\| r(\delta) \kappa(2 \delta)^{3}+2\|\mathcal{E}-\tilde{\mathcal{E}}\|_{\delta} \\
\leq\left\{2^{11} e^{9 K_{j}} \varepsilon+2^{11} e^{7 \delta K_{j}}\right\} \delta\|v\| r(\delta) \kappa(2 \delta)^{3}
\end{gathered}
$$

Consequently, by (III.8),

$$
\begin{aligned}
\left\|\partial \mathcal{A}_{\delta, \varepsilon}(\mathcal{E} ; \cdot)\right\|_{\text {fluct }}+\left\|\partial \mathcal{A}_{\delta, \varepsilon}(\mathcal{E} ; \cdot)-\partial \mathcal{A}_{\delta, \frac{\varepsilon}{2}}(\tilde{\mathcal{E}} ; \cdot)\right\|_{\text {fluct }} \\
\quad \leq\left\{2^{11} e^{9 K_{j}} \varepsilon+2^{12} e^{7 \delta K_{j}}\right\} \delta\|v\| r(\delta) \kappa(2 \delta)^{3} \\
\leq 2^{13} e^{9 K_{j}} \delta\|v\| \| r(\delta) \kappa(2 \delta)^{3} \leq \frac{1}{17}-\frac{1}{32}
\end{aligned}
$$


by (III.1.i) and Hypothesis I.1.iv. Therefore the hypotheses of [3, Corollary 3.6] are satisfied and we have, using (III.3) and (III.12),

$$
\begin{gathered}
\left\|\log \frac{\int d \tilde{\mu}_{r(\delta)}\left(z^{*}, z\right) e^{\partial \mathcal{A}_{\delta, \varepsilon}\left(\mathcal{E} ; \alpha^{*}, \beta ; z_{*}, z\right)}}{\int d \tilde{\mu}_{r(\delta)}\left(z^{*}, z\right)}-\log \frac{\int d \tilde{\mu}_{r(\delta)}\left(z^{*}, z\right) e^{\partial \mathcal{A}_{\delta, \frac{\varepsilon}{2}}\left(\tilde{\mathcal{E}} ; \alpha^{*}, \beta ; z_{*}, z\right)}}{\int d \tilde{\mu}_{r(\delta)}\left(z^{*}, z\right)}\right\|_{2 \delta} \\
\leq 2^{12}\left\{\left\|\partial \mathcal{A}_{\delta, \varepsilon}(\mathcal{E} ; \cdot)\right\|_{\text {fluct }}+\left\|\partial \mathcal{A}_{\delta, \varepsilon}(\mathcal{E} ; \cdot)-\partial \mathcal{A}_{\delta, \frac{\varepsilon}{2}}(\tilde{\mathcal{E}} ; \cdot)\right\|_{\text {fluct }}\right\} \\
\left\|\partial \mathcal{A}_{\delta, \varepsilon}(\mathcal{E} ; \cdot)-\partial \mathcal{A}_{\delta, \frac{\varepsilon}{2}}(\tilde{\mathcal{E}} ; \cdot)\right\|_{\text {fluct }} \\
\leq 2^{25} e^{9 K_{j}} \delta\|v\| r(\delta) \kappa(2 \delta)^{3}\left\{2^{11} e^{9 K_{j}} \varepsilon \delta\|v\| r(\delta) \kappa(2 \delta)^{3}+2\|\mathcal{E}-\tilde{\mathcal{E}}\|_{\delta}\right\}
\end{gathered}
$$

Using this, (III.11) and (III.1.iii), we bound (III.10) by

$$
\left\|\Re_{\delta, \varepsilon}[\mathcal{E}]-\mathfrak{R}_{\delta, \frac{\varepsilon}{2}}[\tilde{\mathcal{E}}]\right\|_{2 \delta} \leq 2^{36} e^{18 K_{j}} \varepsilon \delta^{2}\|v\|^{2} r(\delta)^{2} \kappa(2 \delta)^{6}+q\|\mathcal{E}-\tilde{\mathcal{E}}\|_{\delta}
$$

Corollary III.7 For all sufficiently small $\varepsilon>0$ and integers $0 \leq n \leq \log _{2} \frac{\Theta}{\varepsilon}$, we have

$$
\begin{aligned}
\left\|\mathcal{E}_{\varepsilon_{n}}\left(\varepsilon ; \alpha^{*}, \beta\right)-\mathcal{E}_{\varepsilon_{n}}\left(\frac{\varepsilon}{2} ; \alpha^{*}, \beta\right)\right\|_{\varepsilon_{n}} \leq & K_{E} q^{n} \varepsilon^{2}\|v\| \|^{2} r(\varepsilon)^{2} \kappa(\varepsilon)^{6} \\
& +2^{36} e^{18 K_{j}} \varepsilon\|v\|^{2} \sum_{k=1}^{n} q^{n-k} \varepsilon_{k}^{2} r\left(\varepsilon_{k}\right)^{2} \kappa\left(\varepsilon_{k}\right)^{6}
\end{aligned}
$$

Proof: The proof is by induction on $n$. In the case $n=0, \mathcal{E}_{\varepsilon_{n}}\left(\varepsilon ; \alpha^{*}, \beta\right)=0$ and

$$
\left\|\mathcal{E}_{\varepsilon_{n}}\left(\frac{\varepsilon}{2} ; \alpha^{*}, \beta\right)\right\|_{\varepsilon_{n}} \leq K_{E} \varepsilon^{2}\|v\| \|^{2} r(\varepsilon)^{2} \kappa(\varepsilon)^{6}
$$

by Theorem I.3. For the induction step from $n$ to $n+1$, apply Proposition III.6, with $\delta=\varepsilon_{n}, \mathcal{E}=\mathcal{E}_{\varepsilon_{n}}(\varepsilon)$ and $\tilde{\mathcal{E}}=\mathcal{E}_{\varepsilon_{n}}\left(\frac{\varepsilon}{2}\right)$. This gives, using Hypothesis I.1.1 and the induction hypothesis on $\left\|\mathcal{E}_{\varepsilon_{n}}(\varepsilon)-\mathcal{E}_{\varepsilon_{n}}\left(\frac{\varepsilon}{2}\right)\right\|_{\varepsilon_{n}}$,

$$
\begin{aligned}
& \left\|\mathcal{E}_{\varepsilon_{n+1}}(\varepsilon)-\mathcal{E}_{\varepsilon_{n+1}}\left(\frac{\varepsilon}{2}\right)\right\|_{\varepsilon_{n+1}} \\
& \quad \leq 2^{36} e^{18 K_{j}} \varepsilon\|v\|^{2} \varepsilon_{n}^{2} r\left(\varepsilon_{n}\right)^{2} \kappa\left(\varepsilon_{n+1}\right)^{6}+q\left\|\mathcal{E}_{\varepsilon_{n}}(\varepsilon)-\mathcal{E}_{\varepsilon_{n}}\left(\frac{\varepsilon}{2}\right)\right\|_{\varepsilon_{n}} \\
& \quad \leq K_{E} q^{n+1} \varepsilon^{2}\|v\|^{2} r(\varepsilon)^{2} \kappa(\varepsilon)^{6}+2^{36} e^{18 K_{j}} \varepsilon\|v\|^{2} \sum_{k=1}^{n+1} q^{n+1-k} \varepsilon_{k}^{2} r\left(\varepsilon_{k}\right)^{2} \kappa\left(\varepsilon_{k}\right)^{6}
\end{aligned}
$$


Proof of Theorem I.4: By Corollary III.7, with $\varepsilon=2^{-m} \theta$ and $n=m$, we have, for sufficiently large $m$

$$
\begin{aligned}
\left\|\mathcal{E}_{\theta}\left(\frac{\theta}{2^{m}} ; \alpha^{*}, \beta\right)-\mathcal{E}_{\theta}\left(\frac{\theta}{2^{m+1}} ; \alpha^{*}, \beta\right)\right\|_{\theta} \leq K_{E} \theta^{2}\|v\|^{2}\left(\frac{q}{4}\right)^{m} r\left(\frac{\theta}{2^{m}}\right)^{2} \kappa\left(\frac{\theta}{2^{m}}\right)^{6} & \\
& +2^{36} e^{18 K_{j}}\|v\|^{2} \frac{\theta^{3}}{2^{m}} \sum_{\ell=0}^{m-1}\left(\frac{q}{4}\right)^{\ell} r\left(\frac{\theta}{2^{\ell}}\right)^{2} \kappa\left(\frac{\theta}{2^{\ell}}\right)^{6}
\end{aligned}
$$

and consequently

$$
\begin{aligned}
\sum_{\nu=m}^{\infty} & \left\|\mathcal{E}_{\theta}\left(\frac{\theta}{2^{\nu}} ; \alpha^{*}, \beta\right)-\mathcal{E}_{\theta}\left(\frac{\theta}{2^{\nu+1}} ; \alpha^{*}, \beta\right)\right\|_{\theta} \\
& \leq \operatorname{const} \theta^{2}\|v\|^{2}\left[\sum_{\nu=m}^{\infty}\left(\frac{q}{4}\right)^{\nu} r\left(\frac{\theta}{2^{\nu}}\right)^{2} \kappa\left(\frac{\theta}{2^{\nu}}\right)^{6}+\sum_{\nu=m}^{\infty} \frac{\theta}{2^{\nu}} \sum_{\ell=0}^{\nu-1}\left(\frac{q}{4}\right)^{\ell} r\left(\frac{\theta}{2^{\ell}}\right)^{2} \kappa\left(\frac{\theta}{2^{\ell}}\right)^{6}\right] \\
& \leq \operatorname{const} \theta^{2}\|v\|^{2}\left[\sum_{k=m}^{\infty}\left(\frac{q}{4}\right)^{k} r\left(\frac{\theta}{2^{k}}\right)^{2} \kappa\left(\frac{\theta}{2^{k}}\right)^{6}+\frac{\theta}{2^{m}} \sum_{k=0}^{\infty}\left(\frac{q}{4}\right)^{k} r\left(\frac{\theta}{2^{k}}\right)^{2} \kappa\left(\frac{\theta}{2^{k}}\right)^{6} \sum_{\nu=0}^{\infty} \frac{1}{2^{\nu}}\right]
\end{aligned}
$$

Hence, by Hypothesis I.1.vi and the Cauchy criterion, the sequence $\mathcal{E}_{\theta}\left(2^{-m} \theta ; \alpha^{*}, \beta\right)$ converges uniformly in $\theta$. This gives Theorem I.4. 


\section{Appendix A: Appendix on Stokes' Theorem}

Lemma A.1 Let $r>0$ and $\sigma, \sigma_{*}, \rho \in \mathbb{C}^{X}$ obey $\left|\rho(\mathbf{x})+\sigma_{*}(\mathbf{x})^{*}-\sigma(\mathbf{x})\right|<2 r$ for all $\mathbf{x} \in X$. Set

$$
\begin{aligned}
D_{\sigma_{*}, \sigma, \rho}(\mathbf{x}) & =\left\{\left(z_{*}(\mathbf{x}), z(\mathbf{x})\right) \in \mathbb{C}^{2}|| z_{*}(\mathbf{x})-\sigma_{*}(\mathbf{x})|\leq r,| z(\mathbf{x})-\sigma(\mathbf{x}) \mid \leq r\right. \\
D_{\sigma_{*}, \sigma, \rho} & =\underset{\mathbf{x} \in X}{\mathbf{X}} D_{\sigma_{*}, \sigma, \rho}(\mathbf{x})
\end{aligned}
$$

Let, for each $\mathbf{x} \in X, C_{\sigma_{*}, \sigma, \rho}(\mathbf{x})$ be any two real dimensional submanifold ${ }^{(1)}$ of $\mathbb{C}^{2}$ whose boundary is the union of the one real dimensional submanifolds $\partial D_{\sigma_{*}, \sigma, \rho}(\mathbf{x})$ and the circle $\left\{\left(z_{*}(\mathbf{x}), z(\mathbf{x})\right) \in \mathbb{C}^{2}\left|z_{*}^{*}(\mathbf{x})=z(\mathbf{x}),\right| z(\mathbf{x}) \mid=r\right\}$. Furthermore let $f\left(\alpha_{1}, \cdots, \alpha_{s} ; z_{*}, z\right)$ be a function that is holomorphic in the variables $\alpha_{1}, \cdots, \alpha_{s}$ in a neighbourhood of the origin in $\mathbb{C}^{s X}$ and in the variables $\left(z_{*}, z\right) \in \mathbf{X}_{\mathbf{x} \in X} \mathcal{P}(\mathbf{x})$, with, for each $\mathbf{x} \in X, \mathcal{P}(\mathbf{x})$ being an open polydisc in $\mathbb{C}^{2}$ that contains $C_{\sigma_{*}, \sigma, \rho}(\mathbf{x})$. Then

$$
\begin{aligned}
\int_{D_{\sigma_{*}, \sigma, \rho}} & \prod_{\mathbf{x} \in X}\left[\frac{d z_{*}(\mathbf{x}) \wedge d z(\mathbf{x})}{2 \pi i} e^{-z_{*}(\mathbf{x}) z(\mathbf{x})}\right] e^{f\left(\alpha_{1}, \cdots, \alpha_{s} ; z_{*}, z\right)} \\
= & \sum_{R \subset X} \prod_{\mathbf{x} \in R}\left(\int_{C_{\sigma_{*}, \sigma, \rho}(\mathbf{x})} \frac{d z_{*}(\mathbf{x}) \wedge d z(\mathbf{x})}{2 \pi i} e^{-z_{*}(\mathbf{x}) z(\mathbf{x})}\right) \\
& \left.\prod_{\mathbf{x} \in X \backslash R}\left(\int_{|z(\mathbf{x})| \leq r} \frac{d z(\mathbf{x})^{*} \wedge d z(\mathbf{x})}{2 \pi i} e^{-z_{*}(\mathbf{x}) z(\mathbf{x})}\right) e^{f\left(\alpha_{1}, \cdots, \alpha_{s} ; z_{*}, z\right)}\right|_{\substack{z_{*}(\mathbf{x})=z(\mathbf{x})^{*} \\
\text { for } \mathbf{x} \in \mathbf{X} \backslash \mathrm{R}}}
\end{aligned}
$$

Proof: In the proof we suppress the subscripts $\sigma_{*}, \sigma, \rho$. For each $\mathbf{x} \in X$ there is a three real dimensional submanifold $\mathcal{B}(\mathbf{x}) \subset \mathcal{P}(\mathbf{x})$ whose boundary is the union of $D(\mathbf{x}), C(\mathbf{x})$ and

$$
D_{\mathbb{R}}(\mathbf{x})=\left\{\left(z_{*}(\mathbf{x}), z(\mathbf{x})\right) \in \mathbb{C}^{2}\left|z_{*}^{*}(\mathbf{x})=z(\mathbf{x}),\right| z(\mathbf{x}) \mid \leq r\right\}
$$

We apply Stokes' Theorem once for each point $\mathbf{x} \in X$ to the differential form

$$
\omega=\bigwedge_{\mathbf{x} \in X} \frac{d z_{*}(\mathbf{x}) \wedge d z(\mathbf{x})}{2 \pi i} \exp \left\{-\left\langle z_{*}, z\right\rangle+f\left(\alpha_{1}, \cdots, \alpha_{s} ; z_{*}, z\right)\right\}
$$

Since $\omega$ is a holomorphic $2|X|$ form in $\mathbb{C}^{2|X|}, d \omega=0$ and

$$
\int_{D} \omega=\sum_{R \subset X} \int_{M_{R}} \omega \quad \text { where } \quad M_{R}=\prod_{\mathbf{x} \notin R} D_{\mathbb{R}}(\mathbf{x}) \times \prod_{\mathbf{x} \in R} C(\mathbf{x})
$$

(1) By submanifold, we really mean a submanifold with corners. The orientation of $C_{\sigma_{*}, \sigma, \rho}(\mathbf{x})$ must also be chosen appropriately. 
Example A.2 In Lemma A.1, $C(\mathbf{x})=C_{\sigma_{*}, \sigma, \rho}(\mathbf{x})$ must be a surface whose boundary coincides with the union of the boundaries of $D_{\mathbb{R}}(\mathbf{x})$ and $D_{\sigma_{*}, \sigma, \rho}(\mathbf{x})$. A possible choice of such a surface is constructed as follows. Interpolate between $D_{\mathbb{R}}(\mathbf{x})$ and $D_{\sigma_{*}, \sigma, \rho}(\mathbf{x})$ by the three dimensional set $\mathcal{B}(\mathbf{x})=\bigcup_{0 \leq t \leq 1} D_{t}(\mathbf{x})$ where

$$
D_{t}(\mathbf{x})=\left\{\left(z_{*}, z\right) \in \mathbb{C}^{2}|| z_{*}-t \sigma_{*}(\mathbf{x})|\leq r,| z-t \sigma(\mathbf{x}) \mid \leq r, z-z_{*}^{*}=t \rho(\mathbf{x})\right\}
$$

Then $C(\mathbf{x})=\bigcup_{0<t<1} \partial D_{t}(\mathbf{x})$ has the required boundary.

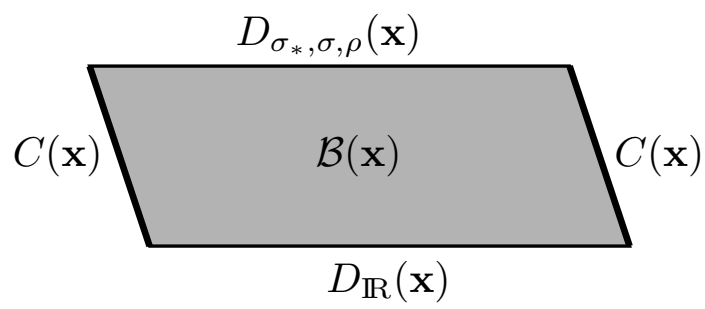

Remark A.3 In the example above,

$$
\operatorname{Re}\left(z_{*} z\right) \geq \frac{1}{2}\left(r^{2}-|\rho(\mathbf{x})|^{2}\right)-r\left(|\sigma(\mathbf{x})|+\left|\sigma_{*}(\mathbf{x})\right|\right)
$$

for all $\left(z_{*}, z\right) \in C(\mathbf{x})$. Furthermore the area of $C(\mathbf{x})$ is bounded by $8 \pi r\left[|\sigma|+\left|\sigma_{*}\right|+|\rho|\right]$.

Proof: Let $\left(z_{*}, z\right) \in C(\mathbf{x})$. We suppress the dependence on $\mathbf{x}$. There is a $0 \leq t \leq 1$ such that $\max \left\{\left|z_{*}-t \sigma_{*}\right|,|z-t \sigma|\right\}=r$ and $z_{*}=z^{*}-t \rho^{*}$. So

$$
\begin{aligned}
& z_{*} z=|z-t \sigma|^{2}+2 \operatorname{Re}(z-t \sigma) t \sigma^{*}+|t \sigma|^{2}-t \rho^{*} z \\
& z_{*} z=\left|z_{*}-t \sigma_{*}\right|^{2}+2 \operatorname{Re}\left(z_{*}-t \sigma_{*}\right) t \sigma_{*}^{*}+\left|t \sigma_{*}\right|^{2}+t \rho z^{*}-|t \rho|^{2}
\end{aligned}
$$

Adding and taking the real part,

$$
\begin{array}{rlr}
2 \operatorname{Re}\left(z_{*} z\right) & =|z-t \sigma|^{2}+\left|z_{*}-t \sigma_{*}\right|^{2}+2 \operatorname{Re}(z-t \sigma) t \sigma^{*}+2 \operatorname{Re}\left(z_{*}-t \sigma_{*}\right) t \sigma_{*}^{*} \\
& +t^{2}\left(|\sigma|^{2}+\left|\sigma_{*}\right|^{2}-|\rho|^{2}\right) \\
& \geq r^{2}-2 r\left(|\sigma|+\left|\sigma_{*}\right|\right)-|\rho|^{2} &
\end{array}
$$

By construction, $C(\mathbf{x})$ is contained in the union of the two cylinders

$$
\begin{aligned}
& \left\{\left(r \zeta+t \sigma, r \zeta^{*}+t\left(\sigma^{*}-\rho^{*}\right)\right)|| \zeta \mid=1, t \in[0,1]\right\} \\
& \left\{\left(r \zeta^{*}+t\left(\sigma_{*}^{*}+\rho\right), r \zeta+t \sigma_{*}\right)|| \zeta \mid=1, t \in[0,1]\right\}
\end{aligned}
$$

The area of the first is bounded by $2 \pi \sqrt{2} r \sqrt{|\sigma|^{2}+|\sigma-\rho|^{2}}$ and the area of the second is bounded by $2 \pi \sqrt{2} r \sqrt{\left|\sigma_{*}\right|^{2}+\left|\sigma_{*}^{*}+\rho\right|^{2}}$. 


\section{Appendix B: Properties of $j(\tau)$}

We discuss the decay properties of the operator $j(\tau)=e^{-\tau(\mathrm{h}-\mu)}$, using the operator norm (I.10).

\section{Lemma B.1}

(a) For any two operators $\mathcal{A}, \mathcal{B}: L^{2}(X) \rightarrow L^{2}(X)$

$$
\|\mathcal{A B}\||\leq\|\mathcal{A}|\||\|\mathcal{B}\||
$$

(b) For any operator $\mathcal{A}: L^{2}(X) \rightarrow L^{2}(X)$ and any complex number $\alpha$

$$
\left\|e^{\alpha \mathcal{A}}\right\| \leq e^{|\alpha|\|\mathcal{A}\|} \quad\|\| e^{\alpha \mathcal{A}}-11\left|\|\leq|\alpha| \mid\| \mathcal{A}\|\| e^{|\alpha|\|\mathcal{A} \mid\|}\right.
$$

Proof: (a) By the triangle inequality, for each $\mathbf{x} \in X$,

$$
\begin{aligned}
\sum_{\mathbf{y} \in X} e^{\mathrm{m} d(\mathbf{x}, \mathbf{y})}|(\mathcal{A B})(\mathbf{x}, \mathbf{y})| & \leq \sum_{\mathbf{y}, \mathbf{z} \in X} e^{\mathrm{m} d(\mathbf{x}, \mathbf{z})}|\mathcal{A}(\mathbf{x}, \mathbf{z})| e^{\mathrm{m} d(\mathbf{z}, \mathbf{y})}|\mathcal{B}(\mathbf{z}, \mathbf{y})| \\
& \leq \sum_{\mathbf{z} \in X} e^{\mathrm{m} d(\mathbf{x}, \mathbf{z})}|\mathcal{A}(\mathbf{x}, \mathbf{z})|\|\mathcal{B}\| \mid \\
& \leq\|\mathcal{A}\|\||\|\mathcal{B}\||
\end{aligned}
$$

The other bound is similar.

(b) By part (a),

$$
\left\|e^{\alpha \mathcal{A}}\right\| \leq \sum_{n=0}^{\infty} \frac{1}{n !}\left\|\left|\alpha^{n} \mathcal{A}^{n}\left\|\leq \sum_{n=0}^{\infty} \frac{1}{n !}|\alpha|^{n}\right\| \mathcal{A}\right|\right\|^{n}=e^{|\alpha|\|\mathcal{A}\| \mid}
$$

and

$$
\left.\left.\left\|e^{\alpha \mathcal{A}}-\mathbb{1}\left|\left\|\leq \sum_{n=1}^{\infty} \frac{1}{n !}\right\|\right| \alpha^{n} \mathcal{A}^{n}\right\|\left|\leq \sum_{n=1}^{\infty} \frac{1}{n !}\right| \alpha\right|^{n}\|\mathcal{A}\|\right|^{n} \leq|\alpha|\|\mathcal{A} \mid\| e^{|\alpha|\|\mathcal{A}\| \|}
$$


Corollary B.2 Let $\tau \geq 0$.

$$
\|j(\tau)\| \leq e^{\tau(\|\mathrm{h}\| \mid+\mu)} \quad\left\||\|j(\tau)-\mathbb{1}\|| \mid \leq \tau(\|\mathrm{h}\||+| \mu \mid) e^{\tau(\|\mathrm{h}\||\|+| \mu \mid)}\right.
$$

Proof: Write $j(\tau)=e^{\tau \mu} e^{-\tau \mathrm{h}}$ and $j(\tau)-\mathbb{1}=e^{\tau \mu}\left(e^{-\tau \mathrm{h}}-\mathbb{1}\right)+e^{\tau \mu}-\mathbb{1}$. By the previous Lemma

$$
\|j(\tau)\| \mid=e^{\tau \mu}\left\|e^{-\tau \mathrm{h}}\right\| \leq e^{\tau \mu} e^{\tau\|\mathrm{h}\| \|}
$$

and

$$
\left\|\left|(\tau)-\mathbb{1}\left\|\left|\leq e^{\tau \mu}\left\|\left|e^{-\tau \mathrm{h}}-\mathbb{1}\right|\right\|+\right|\right\| e^{\tau \mu}-\mathbb{1}\||\leq \tau|\| \mathrm{h} \| e^{\tau \mu} e^{\tau\|\mathrm{h}\| \mid}+\right| e^{\tau \mu}-1 \mid\right.
$$




\section{Appendix C: The Normalization Constant}

We define the normalization constants $\mathcal{Z}_{\delta}(\varepsilon)$ by the recursion relation

$$
\mathcal{Z}_{\varepsilon}(\varepsilon)=1 \quad \mathcal{Z}_{2 \delta}(\varepsilon)=\mathcal{Z}_{\delta}(\varepsilon)^{2} \int_{|z| \leq r(\delta)} \frac{d z^{*} \wedge d z}{2 \pi i} e^{-|z|^{2}}
$$

Lemma C.1 For all $0<\varepsilon<\delta$ with $\frac{\delta}{\varepsilon}$ a positive integer power of 2 , we have $0<\mathcal{Z}_{\delta}(\varepsilon)<1$ and

$$
\left|\ln \mathcal{Z}_{\delta}(\varepsilon)\right| \leq e^{-r(\delta)^{2}}
$$

Furthermore, the limit $\mathcal{Z}_{\delta}=\lim _{n \rightarrow \infty} \mathcal{Z}_{\delta}\left(\frac{\delta}{2^{n}}\right)$ exists and also obeys $\left|\ln \mathcal{Z}_{\delta}\right| \leq e^{-r(\delta)^{2}}$.

Proof: Start by fixing any $\varepsilon>0$ and writing $\varepsilon_{n}=2^{n} \varepsilon$. From the inductive definition,

$$
\ln \mathcal{Z}_{\varepsilon_{n+1}}(\varepsilon)=2 \ln \mathcal{Z}_{\varepsilon_{n}}(\varepsilon)+\ln \mathcal{Z}_{\varepsilon_{n}}^{\prime} \quad \text { where } \mathcal{Z}_{\varepsilon_{n}}^{\prime}=\int_{|z| \leq r\left(\varepsilon_{n}\right)} \frac{d z^{*} \wedge d z}{2 \pi i} e^{-z^{*} z}<1
$$

so that $0<\mathcal{Z}_{\varepsilon_{n}}(\varepsilon)<1$ for all $n \in \mathbb{N}$ and

$$
2^{-n-1}\left|\ln \mathcal{Z}_{\varepsilon_{n+1}}(\varepsilon)\right|=2^{-n}\left|\ln \mathcal{Z}_{\varepsilon_{n}}(\varepsilon)\right|+2^{-n-1}\left|\ln \mathcal{Z}_{\varepsilon_{n}}^{\prime}\right|
$$

which implies

$$
2^{-n}\left|\ln \mathcal{Z}_{\varepsilon_{n}}(\varepsilon)\right|=\sum_{k=0}^{n-1} 2^{-k-1}\left|\ln \mathcal{Z}_{\varepsilon_{k}}^{\prime}\right|
$$

Since

$$
1-\mathcal{Z}_{\varepsilon_{k}}^{\prime}=\int_{|(x, y)| \geq r\left(\varepsilon_{k}\right)} \frac{d x d y}{\pi} e^{-\left(x^{2}+y^{2}\right)}=\frac{1}{\pi} \int_{r\left(\varepsilon_{k}\right)}^{\infty} d r \int_{0}^{2 \pi} d \theta r e^{-r^{2}}=\int_{r\left(\varepsilon_{k}\right)^{2}}^{\infty} d s e^{-s}=e^{-r\left(\varepsilon_{k}\right)^{2}}
$$

and $|\ln (1-x)| \leq \frac{|x|}{1-|x|} \leq 2|x|$ for all $|x| \leq \frac{1}{2}$,

$$
e^{r\left(\varepsilon_{n}\right)^{2}}\left|\ln \mathcal{Z}_{\varepsilon_{n}}(\varepsilon)\right|=\sum_{k=0}^{n-1} 2^{n-k-1} e^{r\left(\varepsilon_{n}\right)^{2}}\left|\ln \left(1-e^{-r\left(\varepsilon_{k}\right)^{2}}\right)\right| \leq \sum_{k=0}^{n-1} 2^{n-k} e^{-\left(r\left(\varepsilon_{k}\right)^{2}-r\left(\varepsilon_{n}\right)^{2}\right)}
$$

By Hypothesis I.1.iii,

$$
\begin{aligned}
r\left(\varepsilon_{k}\right)^{2}-r\left(\varepsilon_{n}\right)^{2} & =\sum_{p=k}^{n-1}\left(r\left(\varepsilon_{p}\right)^{2}-r\left(\varepsilon_{p+1}\right)^{2}\right) \geq \sum_{p=k}^{n-1} r\left(\varepsilon_{p}\right)\left(r\left(\varepsilon_{p}\right)-r\left(\varepsilon_{p+1}\right)\right) \geq \sum_{p=k}^{n-1} 2 \\
& =2(n-k)
\end{aligned}
$$


so that

$$
e^{r\left(\varepsilon_{n}\right)^{2}}\left|\ln \mathcal{Z}_{\varepsilon_{n}}(\varepsilon)\right| \leq \sum_{k=0}^{n-1} 2^{n-k} e^{-2(n-k)} \leq \sum_{\ell=1}^{\infty}\left(\frac{2}{e^{2}}\right)^{\ell}=\frac{2 / e^{2}}{1-2 / e^{2}} \leq 1
$$

For the limit, we rewrite (C.1)

$$
\left|\ln \mathcal{Z}_{\varepsilon_{n}}(\varepsilon)\right|=\sum_{k=0}^{n-1} 2^{n-k-1}\left|\ln \mathcal{Z}_{\varepsilon_{k}}^{\prime}\right|=\sum_{\ell=1}^{n} 2^{\ell-1}\left|\ln \mathcal{Z}_{\varepsilon_{n-\ell}}^{\prime}\right|
$$

which implies

$$
\left|\ln \mathcal{Z}_{\delta}\left(\frac{1}{2^{n}} \delta\right)\right|=\sum_{\ell=1}^{n} 2^{\ell-1}\left|\ln \mathcal{Z}_{2^{-\ell} \delta}^{\prime}\right|
$$

and hence

$$
\begin{aligned}
\lim _{n \rightarrow \infty}\left|\ln \mathcal{Z}_{\delta}\left(\frac{1}{2^{n}} \delta\right)\right| & =\sum_{\ell=1}^{\infty} 2^{\ell-1}\left|\ln \mathcal{Z}_{2^{-\ell} \delta}^{\prime}\right|=\sum_{\ell=1}^{\infty} 2^{\ell-1}\left|\ln \left(1-e^{-r\left(2^{-\ell} \delta\right)^{2}}\right)\right| \\
& \leq e^{-r(\delta)^{2}} \sum_{\ell=1}^{\infty} 2^{\ell} e^{-\left(r\left(2^{-\ell} \delta\right)^{2}-r(\delta)^{2}\right)} \leq e^{-r(\delta)^{2}} \sum_{\ell=1}^{\infty} 2^{\ell} e^{-2 \ell} \\
& \leq e^{-r(\delta)^{2}}
\end{aligned}
$$




\section{Appendix D: The Proof of Example I.2}

Example I.2 Let $\mathfrak{v}>0$.

(i) Suppose that

$$
\kappa(t)=\frac{1}{\sqrt[4]{\|v\| \|}}\left(\frac{1}{t}\right)^{a_{\kappa}} \quad \text { and } \quad r(t)=\frac{1}{\sqrt[4]{\|v\| \|}}\left(\frac{1}{t}\right)^{a_{r}}
$$

for some constants $0<a_{r}<a_{\kappa}$ obeying $3 a_{\kappa}+a_{r}<1$. Then there are constants $K_{E}, \Theta$ and $q$ such that Hypothesis I.1 is fulfilled for all nonzero $v$ with $\|v\| \leq \mathfrak{v}$.

(ii) Suppose that

$$
\kappa(t)=\frac{1}{\sqrt[4]{t\|\| v \| \mid}}\left(\ln \frac{1}{t\|v\| \|}\right)^{b} \quad \text { and } \quad r(t)=\left(\ln \frac{1}{t\|v v\|}\right)^{b}
$$

for some $b \geq 1$. Then there are constants $K_{E}, \Theta$ and $q$ such that Hypothesis I.1 is fulfilled for all nonzero $v$ with $\|v\| \mid \leq \mathfrak{v}$.

Proof: (i) We have

$$
\frac{\kappa(2 t)}{\kappa(t)}=\frac{1}{2^{a_{\kappa}}} \quad \frac{r(2 t)}{r(t)}=\frac{1}{2^{a_{r}}} \quad t \mid\|v\| \| r(t) \kappa(t)^{3}=t^{1-a_{r}-3 a_{\kappa}} \quad \frac{r(t)}{\kappa(t)}=t^{a_{\kappa}-a_{r}}
$$

So part (i) of the Hypothesis is trivially fulfilled if $\frac{1}{\sqrt[4]{\mathfrak{b}}} \min \left\{\frac{1}{\Theta^{a_{r}}}, \frac{1}{\Theta^{a_{k}}}\right\} \geq 1$. Part (ii) of the Hypothesis, namely $e^{t K_{j}} \frac{1}{2^{a_{\kappa}}}+4 t^{a_{\kappa}-a_{r}} \leq 1$, is satisfied provided $e^{\Theta K_{j}} \frac{1}{2^{a_{\kappa}}}+4 \Theta^{a_{\kappa}-a_{r}} \leq 1$, which is the case if $\Theta$ is small enough. Part (iii) of the Hypothesis, namely

$$
r(t)[r(t)-r(2 t)]=\frac{1}{\sqrt{\|v\| \|}} \frac{1}{t^{2 a_{r}}}\left[1-\frac{1}{2^{a_{r}}}\right] \geq 2
$$

is satisfied if $\frac{1}{\Theta^{2 a_{r}}}\left[1-\frac{1}{2^{a_{r}}}\right] \geq 2 \sqrt{\mathfrak{v}}$. Part (iv) of the Hypothesis, namely $t^{1-a_{r}-3 a_{\kappa}} \leq \frac{1}{K_{E}}$, is satisfied provided $\Theta^{1-a_{r}-3 a_{\kappa}} \leq \frac{1}{K_{E}}$. The uniform convergence (for each fixed nonzero $v)$ of

$$
t^{2} \sum_{k=0}^{\infty}\left(\frac{q}{4}\right)^{k} r\left(\frac{t}{2^{k}}\right)^{2} \kappa\left(\frac{t}{2^{k}}\right)^{6}=t^{2-2 a_{r}-6 a_{\kappa}}\|v\|^{-2} \sum_{k=0}^{\infty}\left(\frac{q}{4} 2^{2 a_{r}+6 a_{\kappa}}\right)^{k}
$$

is achieved whenever $q<2^{2\left(1-a_{r}-3 a_{\kappa}\right)}$. Finally, to satisfy part (v), we need

$$
\frac{1}{C\left(\Theta, K_{E}\right)} \frac{2}{q} \leq 2^{4 a_{\kappa}} \leq C\left(\Theta, K_{E}\right) \frac{4}{2^{4 a_{r}}}
$$

or

$$
q \geq \frac{2^{1-4 a_{\kappa}}}{C\left(\Theta, K_{E}\right)} \quad \text { and } \quad 2^{4 a_{\kappa}+4 a_{r}-2} \leq C\left(\Theta, K_{E}\right)
$$


Since $a_{\kappa}+a_{r}<a_{\kappa}+\frac{1}{2} a_{\kappa}+\frac{1}{2} a_{r}=\frac{1}{2}\left(3 a_{\kappa}+a_{r}\right)<\frac{1}{2}$, we have $1-4 a_{\kappa}<2\left(1-a_{r}-3 a_{\kappa}\right)$ and hence $\max \left\{1,2^{1-4 a_{\kappa}}\right\}<2^{2\left(1-a_{r}-3 a_{\kappa}\right)}$. Fix any $q$ obeying $\max \left\{1,2^{1-4 a_{\kappa}}\right\}<q<2^{2\left(1-a_{r}-3 a_{\kappa}\right)}$. Then, pick a $C<1$ sufficiently close to 1 that $q \geq \frac{2^{1-4 a_{\kappa}}}{C}$ and $2^{4 a_{\kappa}+4 a_{r}-2} \leq C$. Then, pick a $K_{E}$ large enough that $1-\frac{2^{33} e^{14 K_{j}}}{K_{E}}>C$. Finally, choose $0<\Theta<1$ that is small enough that $\frac{1}{\sqrt[4]{\mathfrak{v}}} \min \left\{\frac{1}{\Theta^{a_{r}}}, \frac{1}{\Theta^{a_{k}}}\right\} \geq 1, e^{\Theta K_{j}} \frac{1}{2^{a_{\kappa}}}+4 \Theta^{a_{\kappa}-a_{r}} \leq 1, \Theta^{1-a_{r}-3 a_{\kappa}} \leq \frac{1}{K_{E}}, \frac{1}{\Theta^{2 a_{r}}}\left[1-\frac{1}{2^{a_{r}}}\right] \geq 2 \sqrt{\mathfrak{v}}$ and $C\left(\Theta, K_{E}\right) \geq C$.

(ii) Set

$$
C_{\Theta}=\left(1-\frac{\ln 2}{\ln \frac{2}{\Theta \mathfrak{v}}}\right)^{b}
$$

Then, for all $0 \leq t \leq \frac{\Theta}{2}$ and $v$ with $\|v\| \leq \mathfrak{v}$,

$$
\frac{r(2 t)}{r(t)}=\left(1-\frac{\ln 2}{\ln \frac{1}{t\|v\| \|}}\right)^{b} \in\left[C_{\Theta}, 1\right)
$$

so that

$$
\frac{\kappa(2 t)}{\kappa(t)} \in \frac{1}{\sqrt[4]{2}}\left[C_{\Theta}, 1\right) \quad t\|v\| \| r(t) \kappa(t)^{3}=\sqrt[4]{t\|\| v \|}\left(\ln \frac{1}{t\|v\| \|}\right)^{4 b} \quad \frac{r(t)}{\kappa(t)}=\sqrt[4]{t\|v v\|}
$$

The proof now continues as in part (i). 


\section{References}

[1] Tadeusz Balaban, Joel Feldman, Horst Knörrer and Eugene Trubowitz, "A Functional Integral Representation for Many Boson Systems. I: The Partition Function", Annales Henri Poincaré 9, 1229-1273 (2008).

[2] Tadeusz Balaban, Joel Feldman, Horst Knörrer and Eugene Trubowitz, "A Functional Integral Representation for Many Boson Systems. II: Correlation Functions", Annales Henri Poincaré, 9 1275-1307 (2008).

[3] Tadeusz Balaban, Joel Feldman, Horst Knörrer and Eugene Trubowitz, "Power Series Representations for Complex Bosonic Effective Actions", Journal of Mathematical Physics, to appear.

[4] Tadeusz Balaban, Joel Feldman, Horst Knörrer and Eugene Trubowitz, "The Temporal Ultraviolet Limit for Complex Bosonic Many-body Models", Annales Henri Poincaré, to appear.

[5] John W. Negele and Henri Orland, Quantum Many-Particle Systems, Addison-Wesley (1988). 